\title{
A Specification Paradigm for the Design and Implementation of Tangible User Interfaces
}

\author{
ORIT SHAER \\ Computer Science Department, Wellesley College \\ and \\ ROBERT J.K JACOB \\ Computer Science Department, Tufts University
}

\begin{abstract}
Tangible interaction shows promise to significantly enhance computer-mediated support for activities such as learning, problem solving, and design. However, tangible user interfaces are currently considered challenging to design and build. Designers and developers of these interfaces encounter several conceptual, methodological and technical difficulties. Among others, these challenges include: the lack of appropriate interaction abstractions, the shortcomings of current user interface software tools to address continuous and parallel interactions, as well as the excessive effort required to integrate novel input and output technologies. To address these challenges, we propose a specification paradigm for designing and implementing Tangible User Interfaces (TUIs), that enables TUI developers to specify the structure and behavior of a tangible user interface using high-level constructs, which abstract away implementation details. An important benefit of this approach, which is based on User Interface Description Language (UIDL) research, is that these specifications could be automatically or semi-automatically converted into concrete TUI implementations. In addition, such specifications could serve as a common ground for investigating both design and implementation concerns by TUI developers from different disciplines.

Thus, the primary contribution of this paper is a high-level UIDL that provides developers, from different disciplines means for effectively specifying, discussing, and programming, a broad range of tangible user interfaces. There are three distinct elements to this contribution: a visual specification technique that is based on Statecharts and Petri Nets, an XML-compliant language that extends this visual specification technique, as well as a proof-of-concept prototype of a Tangible User Interface Management System (TUIMS) that semi-automatically translates high-level specifications into a program controlling specific target technologies.
\end{abstract}

Categories and Subject Descriptors: D.2.2 [ Design Tools and Techniques]: User Interfaces; H5.2. [ User Interfaces]: UIMS

General Terms: Design, Languages

Additional Key Words and Phrases: Tangible Interaction, User Interface Description Language, User Interface Management System, Tangible User Interfaces

\section{INTRODUCTION}

In the last decade we have seen a wave of Human Computer Interaction (HCI) research aimed at fusing the physical and digital worlds. This work has led to the

Permission to make digital/hard copy of all or part of this material without fee for personal or classroom use provided that the copies are not made or distributed for profit or commercial advantage, the ACM copyright/server notice, the title of the publication, and its date appear, and notice is given that copying is by permission of the ACM, Inc. To copy otherwise, to republish, to post on servers, or to redistribute to lists requires prior specific permission and/or a fee.

(C) 2009 ACM 0000-0000/2009/0000-0001 $\$ 5.00$ 
development of a broad range of systems relying on embedding computation within the physical world. Such systems often employ metaphors that give physical form to digital information and are referred to in the literature as Tangible User Interfaces (TUIs) [Ishii and Ullmer 1997; Hornecker and Buur 2006]. TUIs are designed to take advantage of users' well-entrenched knowledge and skills of interaction with the everyday non-digital world, such as spatial, motor and social skills [Jacob et al. 2008]. By leveraging users' existing skills, TUIs offer the possibility of natural interfaces that are intuitive to use and learn. To date, TUI research shows a potential to enhance the way people interact with and leverage digital information in a variety of application domains including learning, collaborative planning and authoring, and problem solving.

However, although TUIs offer the possibility of interfaces that are easier to learn and use, they are currently more difficult to design and build than traditional interfaces. TUI developers face several conceptual, methodological and technical difficulties including the lack of appropriate interaction abstractions, the shortcomings of current software tools to address continuous and parallel interactions, and the excessive effort required to integrate novel input and output technologies. This may explain why despite their promise, to date, TUIs are still mostly hand built prototypes, designed and implemented in research labs by graduate students.

In this paper, we propose a new paradigm for developing TUIs that is aimed at alleviating the challenges inherent to the design and implementation of TUIs. Rather than using a particular toolkit for programming a TUI, we propose to specify the structure and behavior of a TUI using high-level constructs, which abstract away implementation details. An important benefit of this approach, which is based on User Interface Description Language (UIDL) research [Olsen 1992], is that these specifications could be automatically or semi automatically converted into concrete TUI implementations. In addition, our experience shows that such specifications are a useful common ground for discussing and refining tangible interaction within an interdisciplinary development team. To support this approach, this paper presents TUIML (Tangible User Interface Modeling Language), a high-level UIDL aimed at providing TUI developers from different disciplines means for specifying, discussing and iteratively programming tangible interaction. TUIML consists of a visual specification technique that is based on Statecharts [Harel 1988] and Petri Nets [Petri 1962], and an XML-compliant language. We also present a top-level architecture and a proof-of-concept prototype of a TUIMS that semi-authomatically converts TUIML specifications into concrete interface.

As TUIs evolve from research prototypes into applications for complex domains, carefully studying a TUI prior to its implementation will become crucial for creating functional and usable interfaces. While existing toolkits lower the threshold for implementing TUIs, TUIML provides a comprehensive set of abstractions for specifying, discussing and programming tangible interactions. The design of TUIML is a result of a user-centered design process in which we leveraged our experience building TUIs as well as our experience teaching a Tangible User Interfaces Laboratory course over four years.

This paper is organized as follows: we first summarize findings from our investigation of the design process of TUIs. Next, we present TUIML's visual specifica- 
tion techniques as well as its XML-compliant form. We discuss the results of our evaluation of the language and present a top-level architecture for TUIMS and a prototype. We close with related work.

\subsection{Investigating TUls' Development Process}

To better understand the challenges facing TUI developers, we investigated the development process of TUIs. In addition to an extensive literature review, our own experience building TUIs, provided experiential knowledge. We also taught a graduate-level TUI laboratory course that was structured around a team project, the development of a novel functional TUI prototype. Over the five semester in which we instructed the course (Tufts University, Spring 05, 06, 07, 08 and Summer 07), our students developed 14 new TUIs, some of these TUIs were presented at the Tangible and Embedded Interaction conference (see Figure 1). The course employed heterogeneous implementation technologies including RFID, computer vision, and micro-controllers. The students worked in interdisciplinary teams that included students with backgrounds in Computer Science, Engineering, Arts, Child Development, and Education. From observing our students at work and analyzing their design and implementation artifacts we gathered additional insight into the development process of TUIs. Following, we summarize our findings.
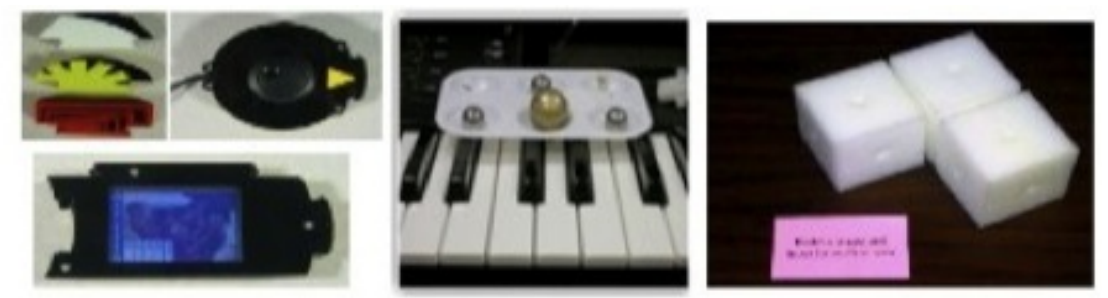

Fig. 1. TVE [Zigelbaum et al. 2007] (left): a TUI for collaborative video editing, implemented with communicating handheld computers. Marble Track Audio Manipulator [Bean et al. 2008] (center): an augmented toy for collaborative music authoring, implemented with an IPAC controller. Smart Blocks [Girouard et al. 2007] (right): computational manipulatives for learning basic geometrical concepts, implemented with RFID.

\subsection{TUI Development Challenges}

We found that TUI developers face a set of conceptual, methodological and technical difficulties throughout the development process of TUIs, as described below. Some of these challenges were preliminary discussed in [Shaer et al. 2004].

1.2.1 Designing an Interplay of Virtual and Physical. As TUIs employ both virtual and physical objects an important role of a TUI developer is to invent metaphors that give physical form to digital information, and to determine which information is best represented digitally and which is best represented physically [Ullmer 2002]. To make physical representations legible, TUI developers are required to consider design issues such as physical syntax [Ullmer 2002], dual feedback loop (digital and physical), perceived coupling (the extent to which the link 
between users actions and systems response is clear) [Hornecker and Buur 2006] and observability (the extent to which the physical state of the system indicate its internal state). However, to date, there are no frameworks or tools that provide vocabulary and means for systematically investigating these issues. Hence, the discussion, comparison and refinement of designs in respect to these issues often performed in an ad-hoc way that does not support concise communication. In our TUI laboratory, a general theme among students was that developing their TUI conceptual design was the most challenging. As one student reported "The biggest difficulties were conceptual. After deciding on the high-level concept, we struggled to refine the interaction techniques".

1.2.2 Selecting from Multiple Behaviors and Actions. While a widget in a GUI encapsulates its behavior, the behavior of a physical object in a TUI is not determined by its nature alone but also by that objects context of use. The behavior of a physical interaction object may change when a new physical object is added to the TUI or when it is physically associated with another physical object. Thus, for each interaction object, a TUI developer is required to define a behavior for each possible context of use. Furthermore, in the physical world there are numerous actions that can be performed with, or upon, any physical object. It is the role of the TUI developer to select and define which actions are meaningful in which context, communicate this information to users, and implement a solution for computationally sensing these actions. However, current tools and interaction models do not provide means for systematically defining a set of alternative behaviors for each physical interaction object.

1.2.3 The Lack of Standard Input and Output Devices. Currently, there are no standard I/O devices for TUIs. Among the technologies for tracking objects, gestures and users in the physical world are RFID, computer vision, microcontrollers and sensors. A variety of actuators are used for creating physical output. In many cases, a TUI is prototyped several times using different technology in each iteration. As each of these technologies currently requires a different set of physical devices and instructions, integrating and customizing novel technologies is difficult and costly. In addition, it is common to implement a particular interaction design by combining or customizing technologies. In our TUI laboratory teams that combined technologies often reported integration difficulties.

1.2.4 Designing Continuous Interaction. When continuously interacting with a TUI, users perceive that there is a persistent connection between a physical object and digital information. However, current tools and techniques do not provide means for exploring continuous interaction. Furthermore, existing event-based software models fail to capture continuous interaction explicitly. Thus, TUI developers are often required to deal with continuous interaction using low-level programming.

1.2.5 Designing Parallel Interaction. In a TUI, multiple users can interact in parallel with multiple physical objects. In addition, a single action may be performed in parallel across multiple physical objects. When designing parallel interaction, TUI developers are required to consider issues such as access points [Hornecker and Buur 2006], spatial and temporal coordination. However, existing 
models usually handle multiple input devices by serializing all input into one common stream [Jacob et al. 1999]. This method is not appropriate for TUIs, since the input is logically parallel. (We refer here to parallel design at the conceptual and software model level, not at the microprocessor level).

1.2.6 Crossing Disciplinary Boundaries. Designing and building a TUI requires cross-disciplinary knowledge. Thus, TUIs are often developed by interdisciplinary teams. While each discipline contributes skills necessary for building TUIs, it also brings different terminologies and work practices. To date, there are no languages and tools that provide TUI developers from different disciplines a common ground for addressing TUI development challenges. In our class, interdisciplinary teams experienced communication and workload division problems. As one student explained "The division of labor problem cropped up partially because we were unable to come up with a clear specification". Another student described "We didnt well document how different parts will come together so this created a challenge when we put things together, communication is definitely the key".

\subsection{Current Tools Supporting the Development of TUls}

Several tools and techniques are currently used by TUI developers to address these challenges. Following we review these tools and techniques and discuss their strengths and limitations.

1.3.1 Sketches, Diagrams and Low-Fidelity Prototypes. Sketches and diagrams dominate the early ideation stages of TUI development. TUI developers use sketches and diagrams for experimenting with high-level concepts such as tangible representations, physical form, and user experience. However, as Blackwell et al. observed, this exploration process is separate from the design of the dynamic behavior and the underlying software structure [Blackwell et al. 2005]. To date, there is no graphical language that provides TUI developers from different disciplines a common ground for exploring both form and function. TUI developers also build low-fidelity prototypes to examine the form and function of a TUI, and to communicate alternative designs within an interdisciplinary development team. However, low-fidelity prototypes often captured the main use scenario, overlooking alternative scenarios and boundary cases.

1.3.2 Storyboards. Storyboarding is a common technique for demonstrating interactive systems behavior [Truong et al. 2006]. However, storyboarding is less effective for describing the behavior of TUIs becuse continuous and parallel interactions are difficult to depict. Rather, TUI developers often use storyboards to depict concepts such as physical surroundings, user motivation, and emotion.

1.3.3 Functional TUI prototyping using toolkits. Several toolkits have emerged to support the implementation of functional TUI prototypes e.g. [Greenberg and Fitchett 2001; Ballagas et al. 2003; Klemmer et al. 2004; Hartmann et al. 2007]. The major advantage of such toolkits is that they lower the threshold for implementing functional TUI prototypes by handling low-level events. However, as most toolkits provide support for specific technology, each time a TUI is prototyped using different technology and hardware, a TUI developer is required to learn new instructions and rewrite code. Furthermore, although toolkits lower the threshold 
for building functional prototypes, they fall short of providing a comprehensive set of abstractions for specifying and discussing tangible interaction.

\subsection{A Specification Paradigm for Designing and Implementing TUls}

Considering the limitations of existing tools and techniques in alleviating TUI development challenges, we propose a specification paradigm for designing and implementing TUIs. Rather than implementing a TUI using a toolkit for a particular hardware technology, we propose that TUI developers would specify the structure and behavior of a TUI using a high-level UIDL, which abstracts away implementation details. An important benefit of this approach, which is based on UIDL research [Olsen 1992], is that these specifications could be automatically or semi automatically converted into different concrete implementations. Thus, addressing the challenge of a lack of standard input and output devices. In addition, such specifications could serve as a common ground for TUI developers from different backgrounds to systematically investigate a variety of design issues. Thus, alleviating the conceptual challenges of designing an interplay of virtual and physical, designing continuous and parallel interactions, as well as mitigating the methodological challenge of crossing disciplinary boundaries. Our first step toward constructing a comprehensive specification paradigm for TUIs was the TAC framework [Shaer et al. 2004] that introduced a set of high-level constructs for describing the structure of TUIs. In this paper, we extend the TAC framework and present TUIML (Tangible User Interface Modeling Language), a UIDL that is capable of describing both the structure and the behavior of TUIs. While TUIML draws upon the constructs introduced by the TAC framework for describing the structure of TUIs, it introduces an interaction model and novel specification techniques for capturing the dynamic behavior of TUIs.

TUIML aims at providing developers from different backgrounds means for specifying, discussing, and iteratively programming TUIs. However, given the complexity and the nature of TUI development challenges, we believe that like software in general, there is no silver bullet [Brooks 1987] to make TUI design and implementation easier. Rather, we suggest combining the use of TUIML with the techniques and tools discussed above to better address TUI development challenges.

\section{TANGIBLE USER INTERFACE MODELING LANGUAGE}

TUIML is a User Interface Description Language for TUIs. In order to offer a language that serves as a common ground for TUI developers from different disciplines in addressing both design and implementation concerns, we implemented TUIML in two forms: a visual language and an XML-compliant language. Following we describe these two implementations of TUIML.

\subsection{A Visual Modeling Language}

TUIML's visual form combines iconic and diagrammatic approaches, thus, drawing from current practices of both user interface and software design: sketching and diagrammatic modeling. TUIML consists of three diagramming techniques for describing the structure and behavior of TUIs in a technology independent manner. To clearly explain the TUIML notation, we have selected Urp [Underkoffler and 
Ishii 1999] as an example because it is one of the most fully developed and known TUI systems. Urp is a TUI for urban planning that allows users to collaboratively manipulate physical building models and tools upon a surface, in order to perform an analysis of shadows, proximities, and wind. When a building model is added to the surface, it casts graphical shadow, corresponding to a solar shadow. The distance between two buildings is measured using a physical distance-measuring tool. Finally, a computational fluid flow simulation is bound to a physical wind tool. By adding this object to the surface, a wind-flow simulation is activated. Changing the physical orientation of the wind tool alters the orientation of the simulated wind. Though additional features are available, this describes the functionality necessary to understand our examples. Figure 2 shows the Urp interface.
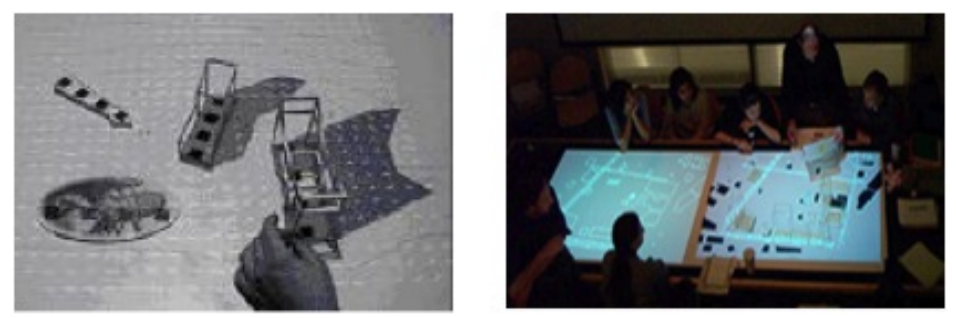

Fig. 2. Urp [Underkoffler and Ishii 1999], a TUI for urban planning.

2.1.1 Describing the Structure of a TUI Using TUIML. To describe the structure of a TUI, TUIML uses a compact set of constructs that includes tokens, constraints and TAC elements. These constructs were defined in the TAC paradigm [Shaer et al. 2004]. Following we introduce a visual notation for these constructs.

A Token is a graspable physical object that represents digital information. A physical object is considered a token only after it is bound to a variable. Users interact with tokens in order to access or manipulate the digital information they represent. The physical properties of a token may reflect the nature of the information it represents. For example, we consider the building models in Urp as tokens as they represent virtual buildings in a computational model. Users interact with physical building models in order to create and alter this model. We also consider the distance-measuring tool and the wind tool as tokens. TUIML depicts tokens using an iconic notation that convey their shape and orientation but abstracts away other physical properties that are specified using a secondary textual notation. Tokens are depicted as simple geometrical shapes that contain a variable. The shapes used to represent a token vary: TUIML offers abstract geometrical shapes, however, a TUI developer may choose to use shapes that resemble the actual look of a certain object. Each shape that is used to represent a token should contain a graphical symbol that represents the variable bound to this token. This additional range of expressiveness have no syntactic meaning thus, TUI developers can freely use customized shapes. By blending informal with formal notation, TUIML supports users from different backgrounds at different stages of the TUI development process. Figure 3, shows TUIML representations of widely used tokens. When a 
TUI contains several instances of the same token type (e.g. eight building models), rather than drawing each separately, TUIML enables to define a token type and then mark the number of token instances using multiplicity indicators.

A Constraint is a physical object that limits the behavior of a token with which it is associated. The physical properties of a constraint guide the user in understanding how to manipulate a token with respect to that constraint and how to interpret configurations of tokens and constraints. For example, in Urp we consider the surface as a constraint because it confines the interaction with building models. It also serves as a reference frame for interpreting the position of building models. Certain physical object may serve as a token, a constraint or both. For example, we consider a building model as a token. However, in the context of measuring distance between two building models, a building model is considered as a constraint because it limits the range of the distance measuring interaction. TUIML depicts constraints using an iconic notation that conveys their shape, orientation and relative size. Again, other physical properties could be specified using a textual secondary notation. Figure 3, shows a TUIML library of widely used constraint types. For each constraint type we define its visual representation, a list of the physical relations possible between this constraint and associated tokens and, a list of user manipulations it supports. TUI developers can easily extend the TUIML notation by adding new constraint types.

A TAC (Token And Constraints) is a relationship between a token and one or more constraints. A TAC relationship often expresses to users something about the kinds of interactions an interface supports. TAC relationships are defined by the TUI developer and are created when a token is physically associated with a constraint. For example, in the Urp system, we consider the configuration of a building model upon a surface as a TAC. Such a TAC is created or modified whenever a building model is added to the surface. Interacting with a TAC involves physically manipulating a token with respect to its constraints. Such interaction has computational interpretation. Manipulation of a token outside its constraints has no computational interpretation. TAC relationships may have a recursive structure so that a given TAC element can serve as a token of another TAC element. Such recursive structure can be used to physically express hierarchical grammars. We view TAC objects as similar to Widgets because they encapsulate the internal state and behavior of physical objects.

To specify the structure of a TUI using TUIML, a TUI developer first describes the set of physical objects employed by a TUI. To define a physical object, a TUI developer provides a name, visual representation and a list of properties. Then, the TUI developer creates a TAC palette, a table that contains all possible TAC relationships of a TUI. Thus, defines a grammar of ways in which objects can be combined together to form meaningful expressions. Such expressions can be interpreted both by users and computational systems. The listing of possible TAC relationships is done in terms of representation, association and manipulation. Representation refers to the binding of a physical object to an application variable to create a token and to the selection of other physical objects that constrain this token. Association refers to the physical association of a token and a set of constraints. Finally, manipulation refers to the actions a user may perform upon a 


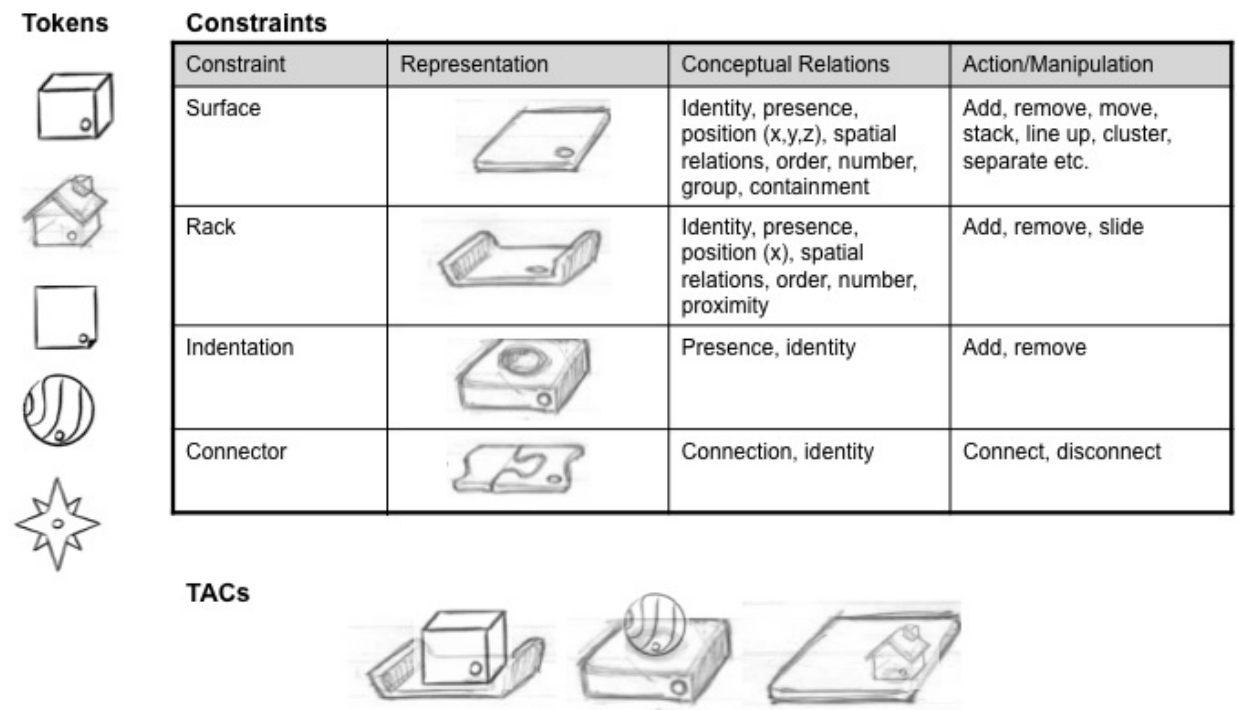

Fig. 3. Graphical representation of tokens, constraints and TAC elements.

TAC. Figure 4 depicts Urp's TAC palette.

The TAC palette allows developers to visually describe the structure of TUIs in terms of tokens, constraints, and TAC elements. It provides TUI developers with means for examining issues such as form, physical syntax, and context of use, that are discussed in the challenges of Designing an Interplay of Virtual and Physical, and Multiple Behaviors and Actions. Also, by providing means for declaring the TAC entities that could exist simultaneously, TUIML addresses aspects related to Parallel Interaction.

2.1.2 Describing Behavior Using TUIML. Current user interface specification languages mainly rely on event-driven models for specifying and programming the current generation of graphical user interfaces. Event-based models are also used for specifying Post-WIMP interfaces and programming discrete 3D and physical interactions [Appert and Beaudouin-Lafon 2006; Hartmann et al. 2006; Wingrave and Bowman 2008]. However, they seem as a wrong model for explicitly specifying continuous and parallel behaviors, which are common in TUIs. Thus, TUIML offers a novel interaction model for describing the underlying behavior of TUIs.

To develop an interaction model that describes the dynamic behavior of a TUI, we examined and studied the dialogue structure and the behavior of a large variety of TUIs. We also analyzed artifacts such as storyboards, natural language descriptions and sketches, all represent common practices for describing TUI behavior. Through this investigation we recognized two fundamental event types that repeat throughout an interaction with a TUI and may cause a mode change in an interface: 1) dynamic binding of digital information to physical interaction objects (i.e. when users couple information to physical objects of their choice) 2) physical 


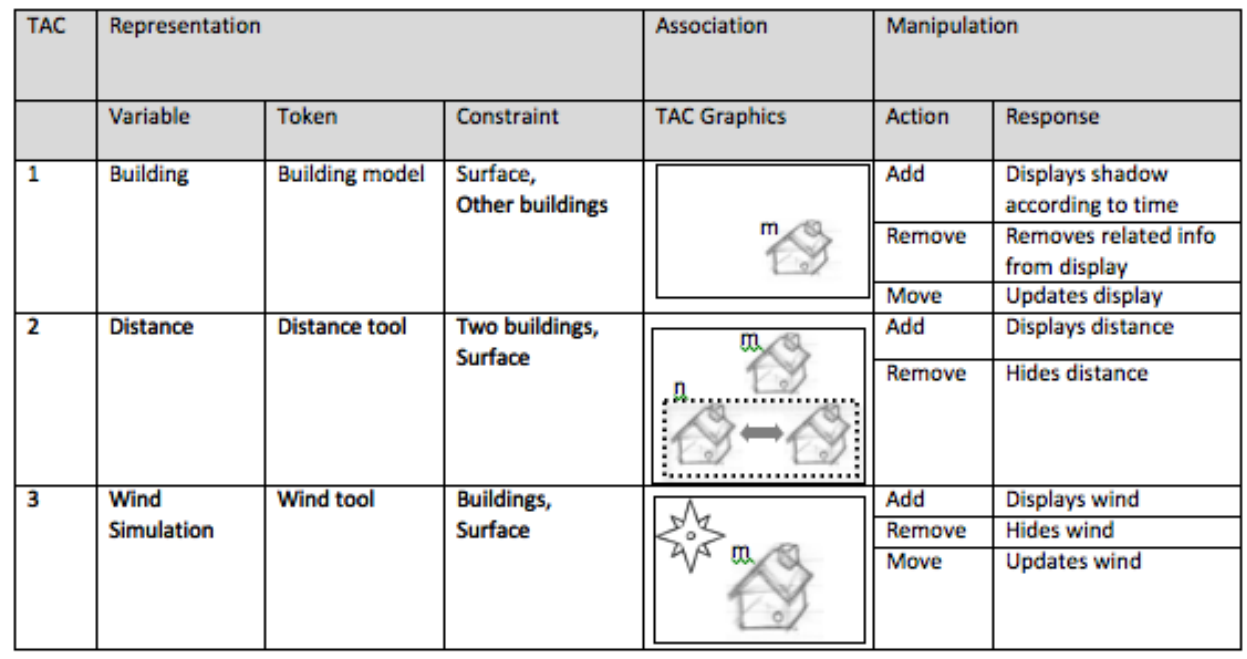

Fig. 4. Urp's [30] TAC palette

association of objects (e.g. when users physically add or connect physical interaction objects to each other). Both event types cause a mode change in an interface because they either alter the meaning of an interaction or modify the range of possible interactions. For example, consider the Urp system, when a second building is added to the surface, the set of possible interactions is modified users can not only add, remove and move a building model but also measure the distance between two buildings. Thus, we identified the basic structure of a tangible dialogue as a sequence of modes or high-level states. However, within each high-level state, multiple users may interact with the system, in a discrete or continuous manner, in parallel or consecutively. Hence, we view the underlying behavior of a TUI as a collection of orthogonal user interactions, each represents a thread of functionality (i.e. task) and is nested within a high-level state.

This leads to a two-tier model for describing the behavior of TUIs. Our two-tier model contains a dialogue tier and an interaction tier (figure 5). The dialogue tier provides an overview of the tangible interaction dialogue and consists of a set of high-level states and transitions. The interaction tier provides a detailed view of each user interaction (i.e. task) that represents a thread of functionality. Following, we introduce a notation for each tier.

The Dialogue Tier. State transition diagram based notations have proven effective and powerful for specifying graphical user interfaces [Olsen 1984; Jacob 1986; Appert and Beaudouin-Lafon 2006] and would be a natural choice for representing the dialogue-tier of our model. However, state diagrams tend to emphasize the modes or states of a system and the sequence of transitions from one state to another, while for TUIs it is important to emphasize that each state contains a set of tasks that could be executed in parallel. Thus, our representation of a dialogue diagram draws upon the Statechart notation [Harel 1988], a state-based notation 


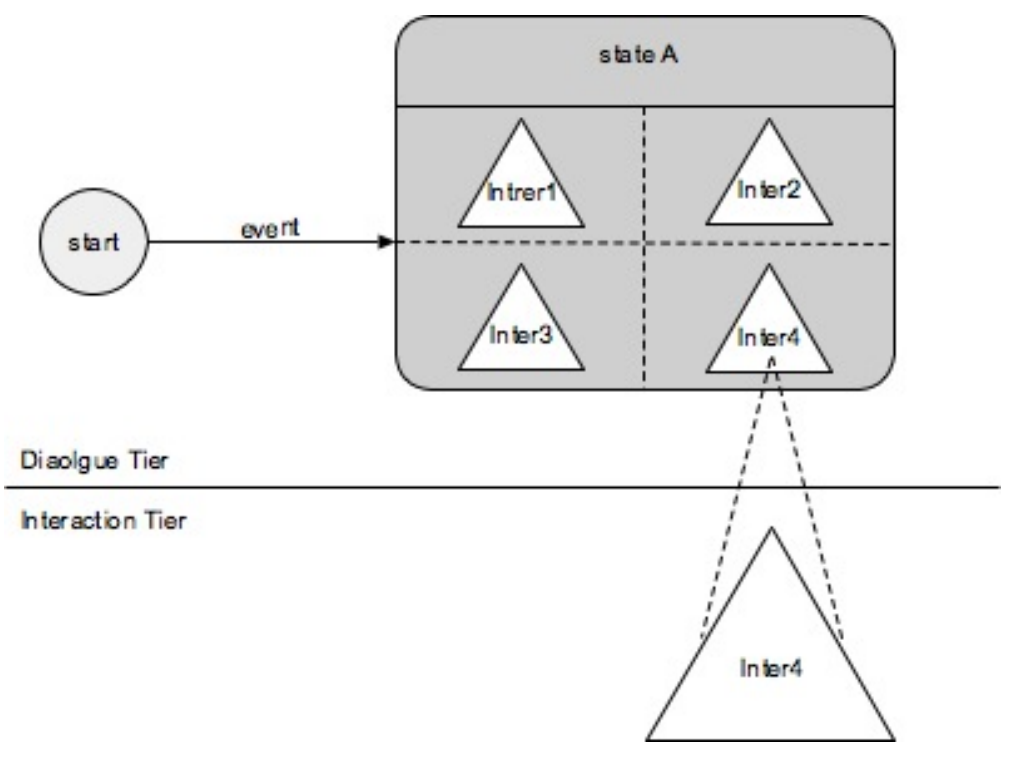

Fig. 5. A two-tier model for describing the behavior of TUIs. The dialogue tier (at the top) describes a set of high-level states a TUI can be in. Each high-level state contains a set of tasks that can be performed when the system is in this state. A zoom in into the dialogue tier reveals the interaction tier: a collection of individual task diagrams nested within each state. Each of these diagrams is represented by a triangle. The internal structure of a task diagram will be explained later in this section.

that enables one to express concurrency within a state. Similarly to Statecharts, TUIML expresses concurrency within a state, but rather than nested concurrent state machines, a TUIML state contains concurrent task diagrams. Task diagrams are based on Petri Nets [Petri 1962] and are designed to capture parallel activities constrained by shared resources. The structure of a task diagram is discussed later in this section.

Rather than describing the state of a TUI at every turn, which would lead to a state explosion, a high-level state captures a context in which certain tasks are available. These tasks are orthogonal and could be performed in parallel assuming their pre-conditions are satisfied. Formally, a high-level state encapsulates three elements: an internal state, a physical state and a set of meaningful interactions. It is denoted by a rounded rectangle and each of the tasks it contains is denoted by a triangle separated from others by a dashed line. Similar to a Statechart [Harel 1988], a dialogue diagram may have initial, final and error states. A transition between high-level states occurs as a result of an event that changes the context in which interactions take place. A transition is associated with an event, a condition and a response. In a TUI, several actors may generate transition events. Thus, TUIML introduces four transition types:timer, system, user interaction, and trigger.

Figure 6, shows the dialogue diagram of the Urp system. The diagram consists 
of three high-level states: an initial state where no building models are located upon the surface, a state where one building model is located upon the surface and a state where at least two building models are located upon the surface. Each of these high-level states contains a set of tasks that users can complete (in sequence or in parallel) while the system is within this state. In the Urp system the transitions from one high-level state to another (or back to the same state) are all a result of user interaction, transitions are associated with a condition (noted as C:) and a response (noted as $\mathrm{R}:)$.

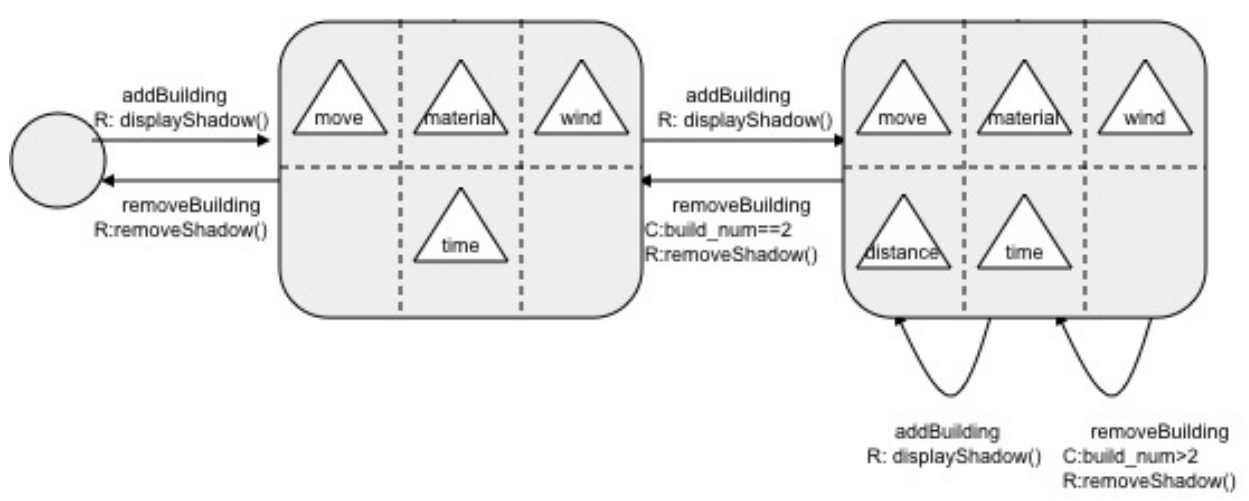

Fig. 6. Urp's dialogue diagram

The Interaction Tier. While the dialogue-tier provides an overview of the tangible dialogue structure, the interaction-tier provides a detailed view of each user interaction that represents a particular task or thread of functionality. A tangible interaction typically consists of a set of discrete actions and continuous manipulations performed consecutively or in parallel upon physical interaction objects. For example, the interaction aimed at distance measuring in the Urp interface consists of a sequence of two discrete user actions: connecting two buildings using a distance-tool (results in displaying the distance between the two buildings) and disconnecting the buildings when the distance display is no longer required. Alternatively, to drive a car down the road users perform two continuous manipulations that are performed in parallel: controlling the steering wheel and adjusting the gas pedal. The interaction-tier depicts this decomposition of tangible interactions into a set of discrete actions and continuous manipulations and specifies the temporal relations between them. For each action or manipulation (discrete or continuous) the diagram specifies an actor, pre-conditions, and post-conditions.

TUIML represents the interaction-tier as a collection of task diagrams each nested within a high-level state. It depicts interaction diagrams using a graphical notation that is inspired from Petri Nets [Petri 1962]) because Petri Nets provides an appealing graphical representation for specifying systems that exhibit parallel activities while being constrained by shared resources. Extensions for Petri nets are used for describing the internal behavior of direct manipulation and 3D interfaces [Bastide

ACM Journal Name, Vol. V, No. N, June 2009. 
and Palanque 1990; Janssen et al. 1993]. However, Petri nets support neither continuous activity nor explicit description of objects. Hence, while our notation draws upon the basic structure of Petri nets to express parallelism, it modifies and enhances the original Petri net notation to produce a graphical notation that explicitly captures these characteristics of tangible interaction.

The basic structure of a task diagram comprises three types of nodes: places, actions and manipulations that are connected by directed arcs. Places represent conditions in terms of physical or digital configurations, actions represent discrete events, and manipulations represent continuous interactions. The diagram is divided into two areas: a physical and a digital world. Figure 7, shows Urp's windsimulation task diagram. Following we further describe the structure this diagram.

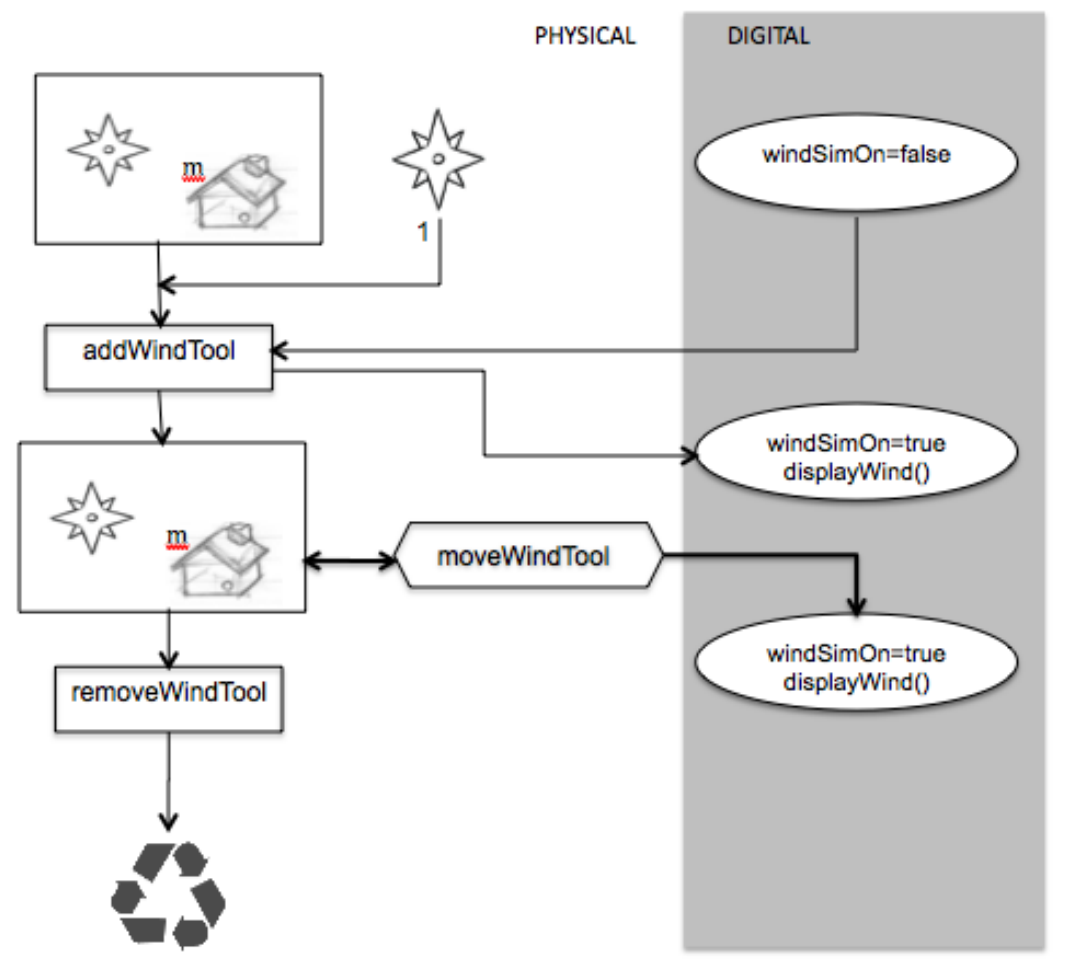

Fig. 7. Urp's wind simulation task diagram. Rectangles depict actions, hexagons depict continuous manipulations.

Places represent pre and post conditions. In a TUI, pre and post conditions may relate to both the physical and the digital states of the system. For example consider the action addWindTool in figure 7 . In order for this action to activate the wind simulation the following pre-conditions must be true: the Urp surface should contain at least one building, the Urp system should contain a wind-tool, but it shouldnt be located upon the surface, the digital flag windSimulationOn should be set to false. TUIML represents places using a mixed notation: physical 
conditions are expressed in terms of tokens, constraints and TACs, digital conditions are represented using text enclosed within an oval node.

Actions are depicted using a box. The actor responsible for generating an action is specified within the box, if no actor is specified, the default actor is the user. The inputs of an action are its pre-conditions, the outputs are its post-conditions. Arcs may be associated with a weight function (i.e. marking) that expresses how many instances of a certain physical interaction object are required for an action to be executed. The wind-simulation task diagram contains two user generated actions: addWindTool and removeWindTool. During the simulation of a task diagram, its initial marking changes according to the sequence of executed actions. Actions that conclude the sequence of executed actions are connected to a special output place called recycler that has no outgoing arcs. Following the execution of such an action, the original marking is recovered.

Manipulations are depicted using a hexagon node. A continuous manipulation last while its pre-conditions hold and may produce continuous digital output. While a manipulation may also produce physical output (e.g. movement), it cannot change the physical state of the system in terms of tokens and constraints configurations. Rather, a continuous manipulation occurs within a particular configuration of tokens and constraints. Thus, places that represent physical conditions serve as both pre and post-conditions of an associated manipulation and are connected to a manipulation node using a thick bi-directional arc.

In the wind-manipulation task diagram, there is one manipulation node, moveWindTool. A pre-condition for this manipulation is a physical configuration, which contains building models and a wind-tool upon a surface. The movement of the windtool upon the surface does not change this physical configuration (it only changes the position of the wind-tool), thus, a bi-directional arc connects the moveWindTool manipulation to the place, which represents this physical configuration. The moveWindTool manipulation causes a continuous update of the digital wind simulation display.

A manipulation may also fire a transition in response to a certain variable crossing a threshold. For example when a user slides an object away from another object, the corresponding manipulation fires an event as a result of the distance between the objects crossing a certain threshold. In such cases, the manipulation node is connected using a regular outgoing flow relation (i.e. an outgoing arc) to a transition that represents the trigger generated event that in turn stops this continuous manipulation. An example for such a manipulation can be found later in the specification of the Tangible Query Interfaces.

A user can stop a continuous manipulation by changing one of its pre-conditions. For example, figure 8 depicts a TUI that consists of a train physical model that moves upon a track. A manipulation tagged moveTrain describes the continuous movement of the train on the track. A user can stop the moveTrain manipulation, by simply removing the train from the track. The system may stop a continuous manipulation by either changing its pre-conditions or by generating an event that directly stops the continuous manipulation. For example, in figure 8, the continuous manipulation moveTrain is generated by the system when a train is placed upon a track if the value of the timer in the digital state of the system equals 0 . Once 


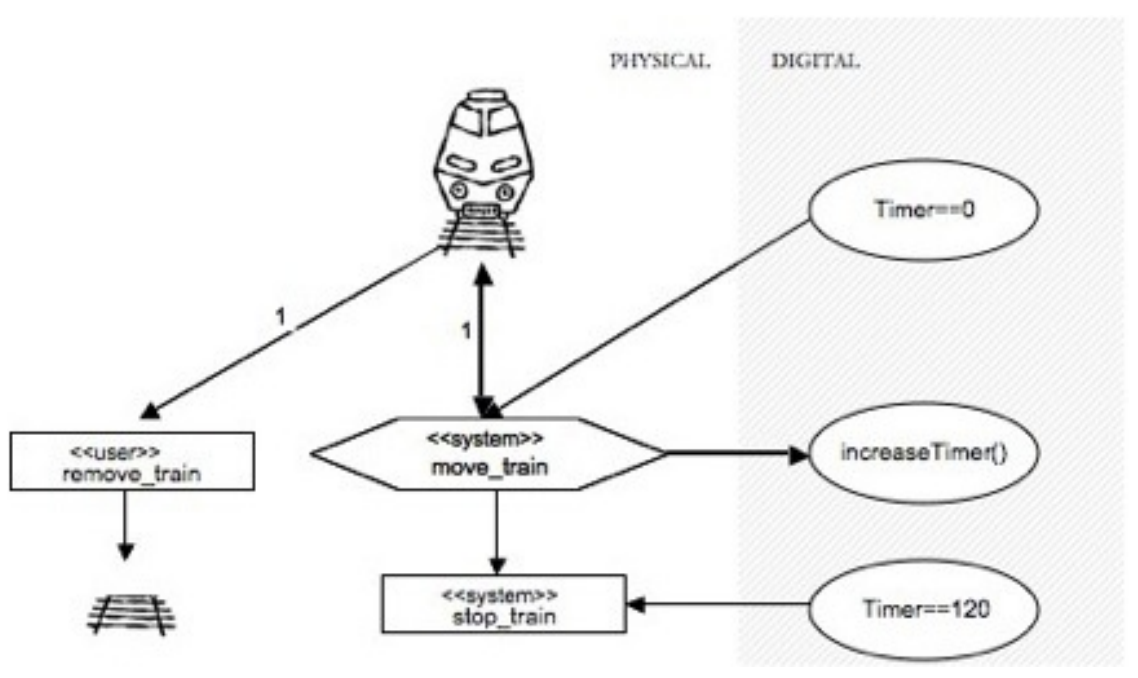

Fig. 8. A Task diagram describing a continous manipulation, move train, that is performed by the system and can be stopped by either the system or the user.

activated, this manipulation continuously increases the timer. When the timer reaches the value of 120 , the system generates an event that stops the train. Note that the moveTrain manipulation is connected to the stopTrain action using a regular outgoing flow relation.

Finally, some tasks contain manipulations or actions that depend on coordination between actors or between the two hands of a single actor (two-hands interaction). Unlike scenarios where two actors perform independent actions simultaneously, we refer here to cases that require actions or manipulations to be temporally and/or spatially coordinated. For example in a video game, flying an airplane requires a user (i.e. pilot) to adjust a speed lever while handling the stick (two-hands interaction), while another user (i.e. navigator) may shoot the enemy. By allowing manipulation and action nodes to serve as pre and post conditions of other action or manipulation nodes, TUIML enables the specification of temporally and/or spatially coordinated actions and manipulations.

2.1.3 Summary. TUIML is aimed at specifying, analyzing and refining tangible interaction. To accomplish these goals it provides means for visualizing the structure and behavior of TUIs. TUIML defines three types of diagrams: The first, the TAC palette, represents the structure of a TUI, the other two diagrams types, the dialogue diagram and the task diagram represent its dynamic behavior.

Visually specifying the structure of a TUI in terms of tokens, constraints, and TAC elements, provides TUI developers with means for examining issues such as form, physical syntax and context of use. The two-tier model, and the dialogue and task diagrams presented here are intended to enable TUI developers to describe and discuss a TUI's behavior from a point of view closer to the user rather than to the exigencies of implementation. The dialogue diagram aims to bring out the big picture, and assist TUI developers to assure that functionality is complete 
and correct prior to implementation. The task diagram allows TUI developers to focus on a specific thread of functionality and provides means for addressing design concerns related to parallel interaction, dual digital and physical feedback loops, and combined discrete and continuous inputs. The comparison of task diagrams allows TUI developers to consider alternative designs. We intend that applying TUIML with a high level of formality could also yield a detailed specification document that can serve to guide implementation. Following we describe an XML-compliant form of TUIML, that formalizes TUIML specifications.

\subsection{TUI Markup Language}

In addition to the visual form of TUIML, we defined an XML-compliant form for TUIML. This XML-compliant form is intended as an intermediate language for a Tangible User Interface Management System (TUIMS) that compiles TUIML specifications to code, and for interoperating with other user interface software tools. It also serves as a secondary notation for the visual form of TUIML, allowing users to specify properties of tokens and constraints as well as to specify which token and TAC relationships are created at design time and which at run-time. While the XML-compliant form of TUIML was not intended directly for user input, it is reasonably human readable and is supported by existing parsing and editing tools. We used the grammar-based XML schema mechanism to formalize the structure and content of TUIML documents.

A TUIML document is composed of two parts: a prolog identifying the XML language version and encoding, and the root element, which is the tuiml tag. The tuiml element contains three child elements: an optional header element that provides metadata about the document, an element that identifies the application logic, and an optional interface description, which describes the structure and the behavior of the interface. The interface element is the heart of the TUIML document. All of the elements that describe the user interface are present within this tag. Figure 9 , depicts the basic structure of the interface element.

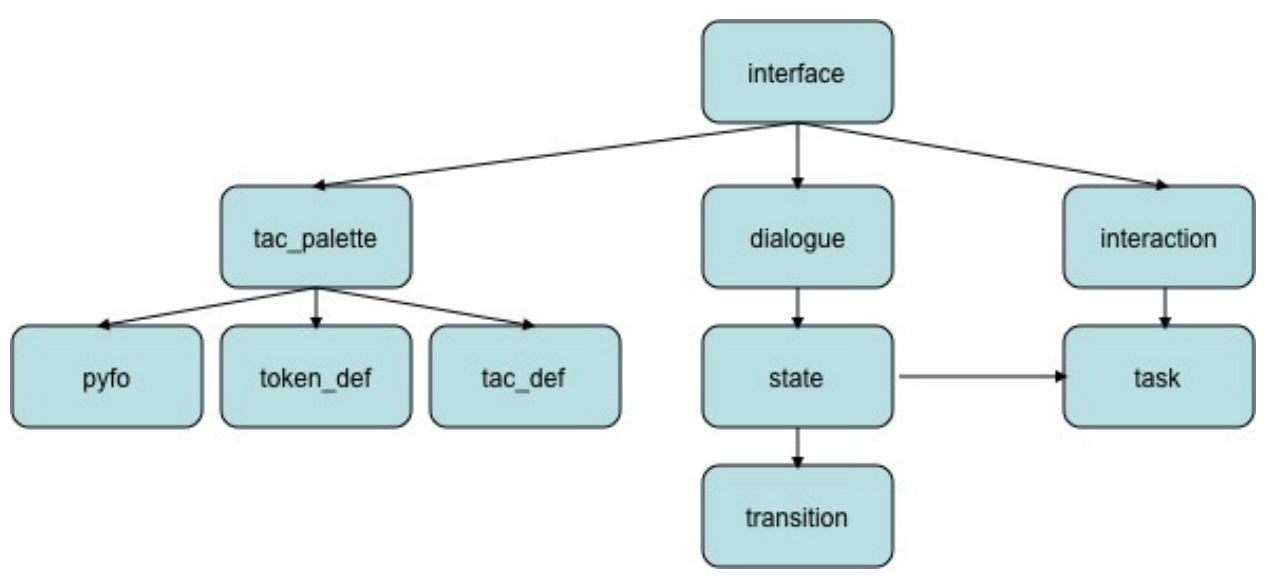

Fig. 9. The top-level elements of TUIml. 
The tac_palette element describes the structure of a TUI in terms of physical interaction objects, tokens, and TAC relations. This element begins with an enumeration of physical interaction objects (i.e. pyfos) that are not bound to digital information. The tac_palette element then lists tokens using token_def element. An interesting aspect of TUIs is that tokens can be either statically bound to digital information by the designer of the system, or dynamically bound by the user at run-time. When a token is statically bound, its token_def element contains a unique name and an application variable. When a token is dynamically bound its token_def element contains a unique class name and a variable type. In addition a token_def element may contain property elements that describe the physical properties of the token. Similiarly to a token a TAC entity could be statically or dynamically created. The corresponding tac_def element of a statically created TAC is given a unique name. The corresponding tac_def element of a dynamically created TAC is given a unique class name.

The dialogue element consists of a sequence of state elements. Each state is given a unique id and contains the following elements: internal_state,physical_state, a collection of task elements, and a collection of transition elements. The internal_state element describes the value of relevant application variables. The physical_state element describes the physical configuration of the system in terms of token and TAC relations. Task elements represent the interaction tasks a user can perform while the system is within this particular state. Finally, a transition element may contain a condition element that must be true in order for the transition to fire, a response element and a tuiml_event element that maps the high-level transition event to a TUIML event that could later be mapped into a low-level event. TUIML events include tokenAdded, tokenRemoved, tokenUpdated and tokenPropertyChanged.

The interaction element consists of a sequence of task elements. Each task element represents a task diagram of a different user interaction or thread of functionality. In defining the structure of a task element we drew upon the Petri Net Markup Language [Weber and Kindler 2003]. A task element consists of the following elements: physical_place, digital_place, recycler, action, manipulation and arc. Each of these elements could be omitted or repeated as needed.

\subsection{TUIML Modeling Tools}

We developed three prototypes of a visual modeling environment for TUIML. These prototypes served as working models for investigating the transformation of visual TUIML specifications to XML-compliant form, as well as for eliciting requirements for a complete modeling environment for TUIML. The first prototype shielded users from the underlying TUIML syntax by allowing users to sketch 3D interaction objects and bind them to behavior using forms. The second prototype, provided a visual editor for TUIML diagrams, it allowed users to create, modify, and save diagrams using a set of pre-defined shapes and tools, users could also load new shapes to be used in their diagrams. Our most recent prototype extended this visual editor and implemented it an Eclipse plug-in. It enabled to visually manipulate TUIML objects and easily connect them to application logic. Our findings from developing and informally evaluating these prototypes played an important role in refining the design of TUIML. 


\subsection{Scope and Limitations}

TUIML, was mostly designed to specify data-centerd TUIs [Hornecker and Buur 2006], a design perspective that is primarily pursued within HCI and Computer Science and results in systems that use spatially configurable physical artifacts as representations and controls for digital information [Ullmer 2002]. Examples of data-centered TUIs that are directly addressed by TUIML include Interactive Surfaces, Constructive Assemblies and Token+Constraints [Ullmer 2002]. TUIML can also be used to specify certain aspects of other TUI design perspective such as the space-centered [Hornecker and Buur 2006] view that focuses on the design of interactive spaces and the expressive-movement-centered view [Hornecker and Buur 2006], that emphasizes expressive movement. However, currently TUIML does not provide means for specifying unique characteristics of these design perspectives such as expressive gestures. Also, several research areas are closely related to TUIs. These include Tangible Augmented Reality, Tangible Table-Top Interaction, and Ambient Displays. TUIML provides means for describing the structure and behavior of such interfaces by enabling the specification of body parts as tokens or constraints, of discrete and continuous touch-based interactions, and of implicit interaction. Finally, several emerging interaction styles, including touch-based interaction, ubiquitous computing, embodied Interaction [?] ,and mixed reality, share salient commonalities with TUIs [Jacob et al. 2008]. Thus, TUIML can describe certain aspects of these interaction styles, but it does not provide means for comprehensively specifying these interaction styles. Finally, TUIML is not intended as a generative design tools, rather it aims at specifying, discussing and iteratively programming tangible interaction.

\section{EVALUATION}

Our evaluation of TUIML focuses on three desirable properties: High ceiling [Myers et al. 2000], the ability to describe a broad range of TUIs. Low threshold [Myers et al. 2000], the extent to which the language is easy to learn and use. Utility and applicability, the ability to alleviate development challenges, and to be applied without excessive effort. We employed two evaluation methods in concert: specification of benchmark TUIs, and analysis of use by students.

\subsection{Specifying Benchmark TUls using TUIML}

Using benchmarks is a known evaluation process in some areas of Human-Computer Interaction (HCI) such as information visualization [Plaisant 2004]. In this section, we report on a benchmark that we have created for the purpose of evaluating the TUIML notation. The benchmark consists of a set of TUIs that are considered the state-of-the-art in the field of tangible interaction. We have chosen to include in the benchmark TUIs that serve as representatives of a larger class of TUIs, so together they cover an important and large subset of the TUI design space. In our selection of TUI classes we utilized Ullmers division of the TUI design space into three high level classifications [Ullmer 2002] and selected representatives from each classification: Interactive Surfaces, Constructive Assemblies, and Token + Constraint. We also selected mainly interfaces that were fully developed, and evaluated. Table 1, lists the nine interfaces that we selected as benchmarks. Each of these interfaces

ACM Journal Name, Vol. V, No. N, June 2009. 
was specified several times throughout the iterative development of TUIML. In addition to the specifications made by the authors, two graduate students specified each of these interfaces. Following, we discuss the TUIML specifications of three benchmark interfaces. By specifying benchmark interfaces we evaluated not only the languages ability to describe a broad range of interfaces in a precise, consistent and simple way, but also the usefulness of the specifications to TUI developers. We looked at what can be learned from these specifications and whether they highlight aspects of tangible interaction such as the use of physical syntax, mapping between shape and function, parallel and continuous interaction.

Table I. Benchmark TUIs

\begin{tabular}{|l|l|}
\hline Interface & TUI category \\
\hline Urp [Underkoffler and Ishii 1999] & Interactive Surfaces \\
\hline Designers' Outpost [Klemmer et al. 2001] & Interactive Surfaces \\
\hline Senseboard [Jacob et al. 2002] & Interactive Surfaces \\
\hline TVE [Zigelbaum et al. 2007] & Constructive Assemblies \\
\hline Navigational Blocks [Camarata et al. 2002] & Constructive Assemblies \\
\hline Tern [Horn and Jacob 2007] & Constructive Assemblies \\
\hline Marble Answering Machine [Crampton-Smith 1995] & Tokens+Constraints \\
\hline Tangible Query Interfaces [Ullmer 2002] & Tokens+Constraints \\
\hline Media Blocks [Ullmer 2002] & Tokens+Constraints \\
\hline
\end{tabular}

3.1.1 The Marble Answering Machine. One of the earliest illustrations of interlinking the physical and digital worlds is provided in the design of the Marble Answering Machine (MAM) [Crampton-Smith 1995]. It was designed and prototyped by Durrell Bishop, while a student at the Royal College of Art, in order to explore ways in which computing can be taken off the desk and integrated into every day objects. Although it was never fully implemented, it is a well-known, influential TUI design that inspired numerous TUI researchers and developers. The MAM system is a simple and elegant example of a token+constraint TUI [Ullmer 2002]. In the Marble Answering Machine, marbles represent incoming voice messages. To play a message, a user grabs a message (marble) and places it in an indentation on the machine. To return a call, the user places the marble within an indentation in an augmented telephone. To store a message, the user places a marble in a dedicated storage saucer different users may have different saucers. Figure 11, illustrates a design sketch of the Marble Answering Machine.

Figure 12, presents the TAC palette for the MAM. Visually specifying the MAM structure highlights the use of physical constraints to enforce physical syntax. For example, TAC 2, consists of a marble and a replay indentation. The shape of the replay indentation affords the placement of a single marble within its dimensions. The visual specification of a TAC could also assist in comparing alternative designs. For example, within the scope of the MAM, we can compare the structure of TAC 1 , a marble within a message queue, with the structure of TAC 4, a marble within a storage saucer. While the physical properties of a rack (used for representing the message queue) imply the following relations: presence, order, and number, the physical properties of a storage saucer only imply the relations of presence and 


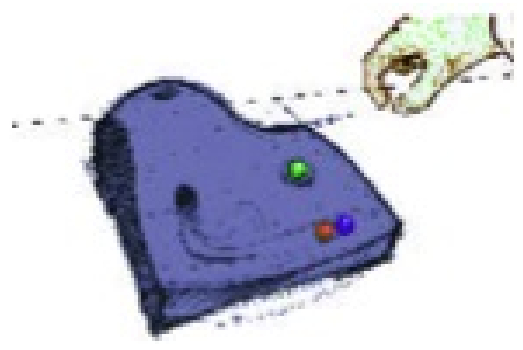

Fig. 10. A design sketch of the Marble Answering Machine, from [Crampton-Smith 1995]. Incoming messages await while the user listens to a message.

number. As such, a user approaching his storage saucer is not aware to the order in which his incoming messages have arrived. Replacing the storage saucer with other constraint types provided by TUIML, such as a rack or a series of indentations allows the TUI developer to consider alternative designs.

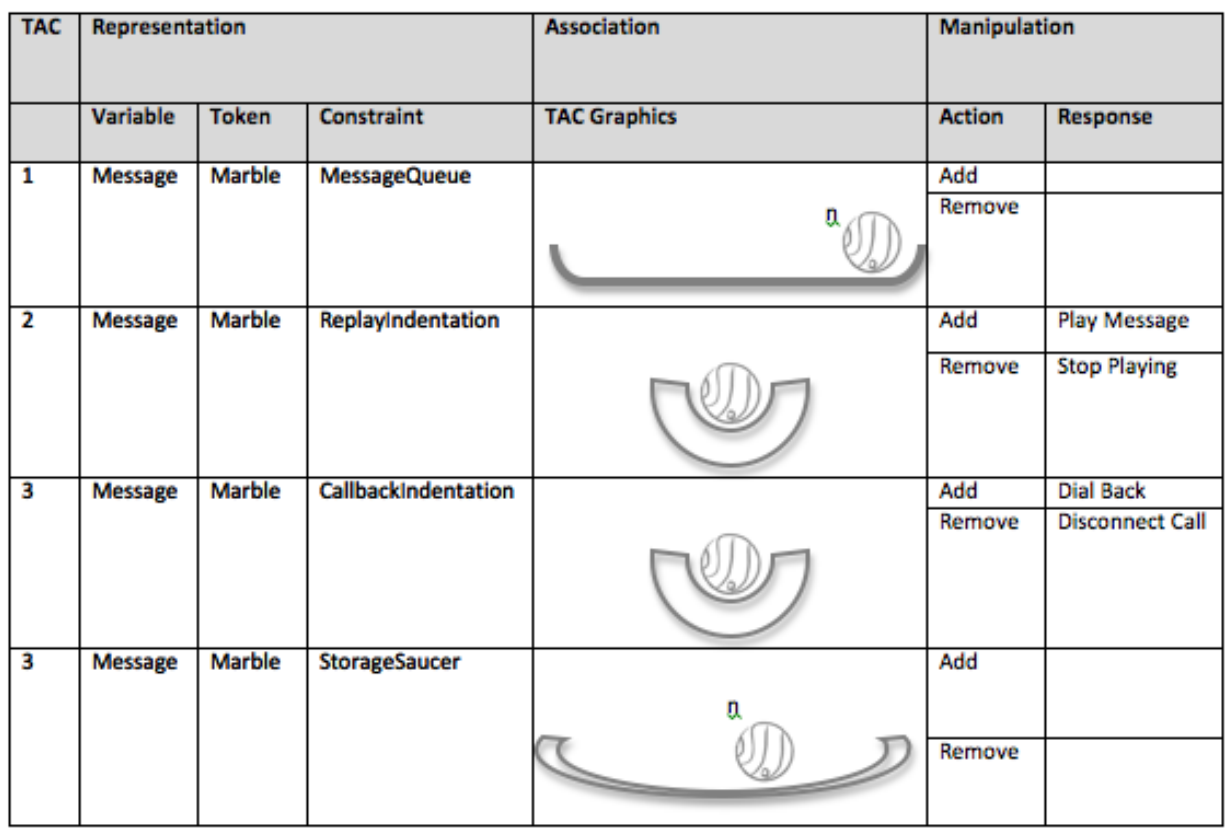

Fig. 11. The Marble Answering Machine's [Crampton-Smith 1995] TAC palette.

Figure 13, presents the dialogue diagram of the MAM interface. This diagram depicts two different transitions types: those generated by system events (e.g. incoming calls) and those generated by users (e.g. remove marble). It is important to note that the description of interactions such as loading the machine is absent from the original description of the system at [Crampton-Smith 1995]).

ACM Journal Name, Vol. V, No. N, June 2009. 


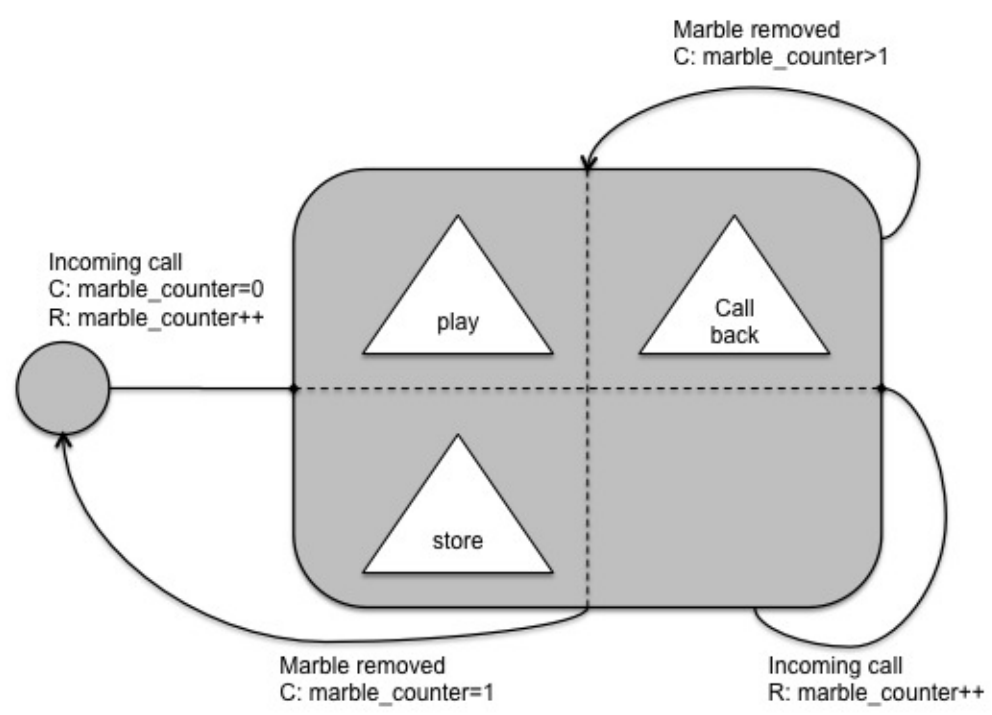

Fig. 12. The Marble Answering Machine‘s [Crampton-Smith 1995] dialogue diagram.

Comparing the play and call back interaction diagrams (Figure 14), highlights a consistent interaction syntax across these two interactions. Also, from considering the interaction objects required for completing each of those interactions (a marble and a play indentation for playing, a message and a marble and a call back indentation for calling back) we learn that these two interactions could take place in parallel assuming the MAM contains at least two different messages.

3.1.2 Navigational Blocks. Navigational Blocks [Camarata et al. 2002] (see figure 15) is a TUI for information space exploration. It consists of four blocks, each represents a major category of historical data. Each face of these blocks is bound to a different instance of this category. To explore the historical information, users interact with these blocks within an active space. Placing a block within the active space displays information from the block category, for example, locating the who block within the active space displays a list of historical figures. Rotating a block within the active space, queries the applications database and displays the information related to the topic represented by the upper face of the block. Translating a block (i.e. sliding), within the active space, scrolls through the displayed information. In addition, users may explore the relationships between two information categories by attaching two blocks together to express a boolean AND. If two topics (those represented by the upper faces of the blocks) are related the blocks attract each other, if these topics are not related the two blocks repel each other. Locating the attached blocks within the active space displays the results of an AND query. The Navigational Blocks system can be viewed as a constructive assembly TUI [Ullmer 2002] because it allows users to create computational expressions by attaching blocks.

Figure 16, depicts the TAC palette of the Navigational Blocks system. It is inter- 


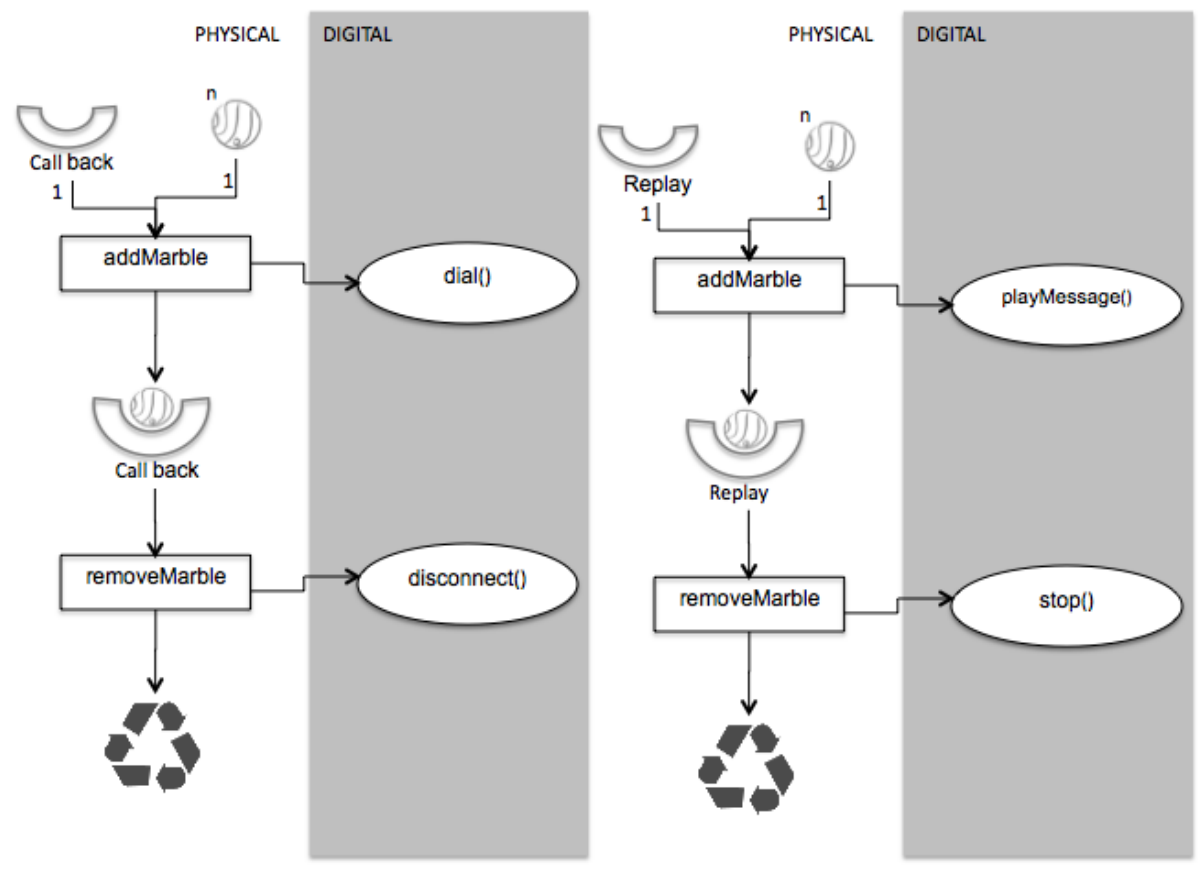

Fig. 13. The Marble Answering Machine's [Crampton-Smith 1995] Call (left) and Play (right) task diagrams.

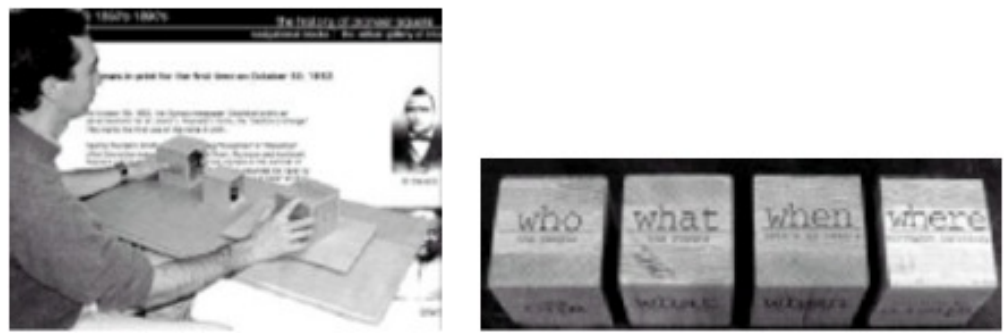

Fig. 14. Navigational Blocks [Camarata et al. 2002]

esting to note, that while the MAM interface [Crampton-Smith 1995] uses physical constraints with one degree of freedom (1DOF) to enforce digital syntax, the Navigational Blocks system constrains the tangible interaction to the dimensions of an active space (6DOF). By choosing so, the Navigational Blocks system provides users with less guidance regarding which possible interactions are meaningful but further encourages users to explore the system. Thus, users may try interacting with the system in several ways, some of them meaningful and defined by the system designer (e.g. adding a block to the active space) while others (e.g. adding several un-attached blocks to the active space) may result in an error. The TAC palette

ACM Journal Name, Vol. V, No. N, June 2009. 
provides TUI developers with a systematic way for defining those manipulations that are meaningful, and identifying manipulations that are probable but are not meaningful.

\begin{tabular}{|c|c|c|c|c|c|c|}
\hline \multirow[t]{2}{*}{ TAC } & \multicolumn{3}{|c|}{ Representation } & \multirow{2}{*}{$\begin{array}{l}\text { Association } \\
\text { TAC Graphics }\end{array}$} & \multicolumn{2}{|c|}{ Manipulation } \\
\hline & Variable & Token & Constraint & & Action & Response \\
\hline \multirow[t]{4}{*}{1} & \multirow[t]{4}{*}{$\begin{array}{l}\text { Information } \\
\text { category }\end{array}$} & \multirow[t]{4}{*}{ Block } & \multirow[t]{4}{*}{ Active space } & \multirow{4}{*}{0} & Add & $\begin{array}{l}\text { Displays information according to top } \\
\text { face. }\end{array}$ \\
\hline & & & & & Remove & Clears display \\
\hline & & & & & Rotate & $\begin{array}{l}\text { Updates displayed information } \\
\text { according to top face. }\end{array}$ \\
\hline & & & & & Slide & $\begin{array}{l}\text { Moves back and forward in } \\
\text { information space. }\end{array}$ \\
\hline \multirow[t]{3}{*}{2} & \multirow[t]{3}{*}{$\begin{array}{l}\text { Query } \\
\text { parameter }\end{array}$} & \multirow[t]{3}{*}{ Block } & \multirow[t]{3}{*}{ Block } & & Attach & $\begin{array}{l}\text { Computes query, attract block if query } \\
\text { result is not empty, repel blocks if } \\
\text { query result is empty. }\end{array}$ \\
\hline & & & & & Rotate & $\begin{array}{l}\text { Updates query result, attract block if } \\
\text { query result is not empty, repel blocks } \\
\text { if query result is empty. }\end{array}$ \\
\hline & & & & & Detach & Undefined. \\
\hline \multirow[t]{4}{*}{3} & \multirow[t]{4}{*}{ AND query } & \multirow{4}{*}{$\begin{array}{l}\text { Two } \\
\text { attached } \\
\text { blocks }\end{array}$} & \multirow[t]{4}{*}{ Active space } & \multirow{4}{*}{$\left.\right|_{0} ^{\pi}$} & Add & Displays query results. \\
\hline & & & & & Rotate & $\begin{array}{l}\text { Updates query result, attract block if } \\
\text { query result is not empty, repel blocks } \\
\text { if query result is empty. }\end{array}$ \\
\hline & & & & & Detach & Undefined| \\
\hline & & & & & Remove & Clears display. \\
\hline
\end{tabular}

Fig. 15. Navigational Blocks' [Camarata et al. 2002] TAC palette.

Figure 17, depicts the Navigational Blocks dialogue diagram. It contains four high-level states: an initial states in which no blocks are present within the interactive space, a state in which one block is present within the interactive space, a state where two attached blocks are present within the interactive space, and an error state. Constructing this diagram highlights some use cases that are not specified in (Camarata et al., 2002) (e.g. the presence of two or more un-attached blocks within the active space).

Figure 18, depicts the task diagram describing the rotation of a block that is part of two attached blocks (i.e. an AND query). It is interesting to note that the Navigational Blocks system could respond in one of two ways to the rotation of a block that is already attached to another if the two topics represented by the blocks top faces produce non empty query results the blocks attract each other otherwise they repel each other. Therefore, the rotate action in figure 18 has two possible physical outputs, This is an example of active tokens that provide physical feedback attraction or repulsion. Currently, due to technical challenges only a few systems employ active tokens, however, in the future we anticipate that TUIs will often provide physical feedback to physical input. 


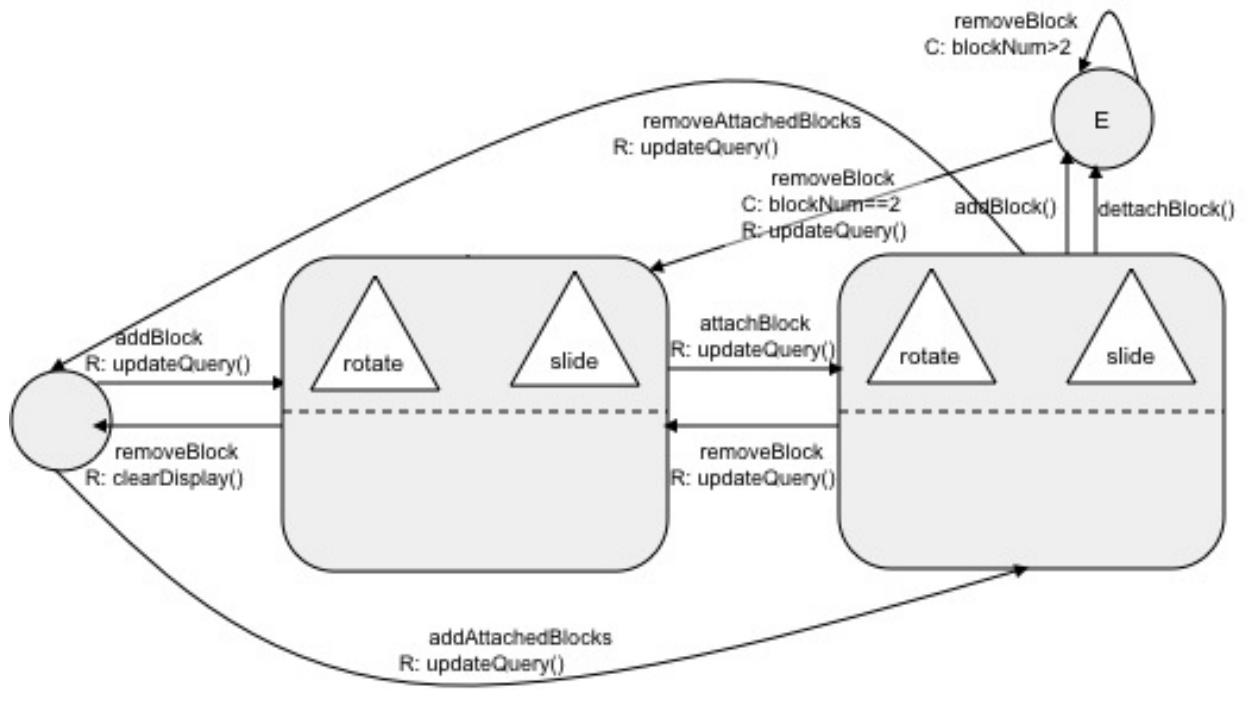

Fig. 16. Navigational Blocks' [Camarata et al. 2002] dialogue diagram.

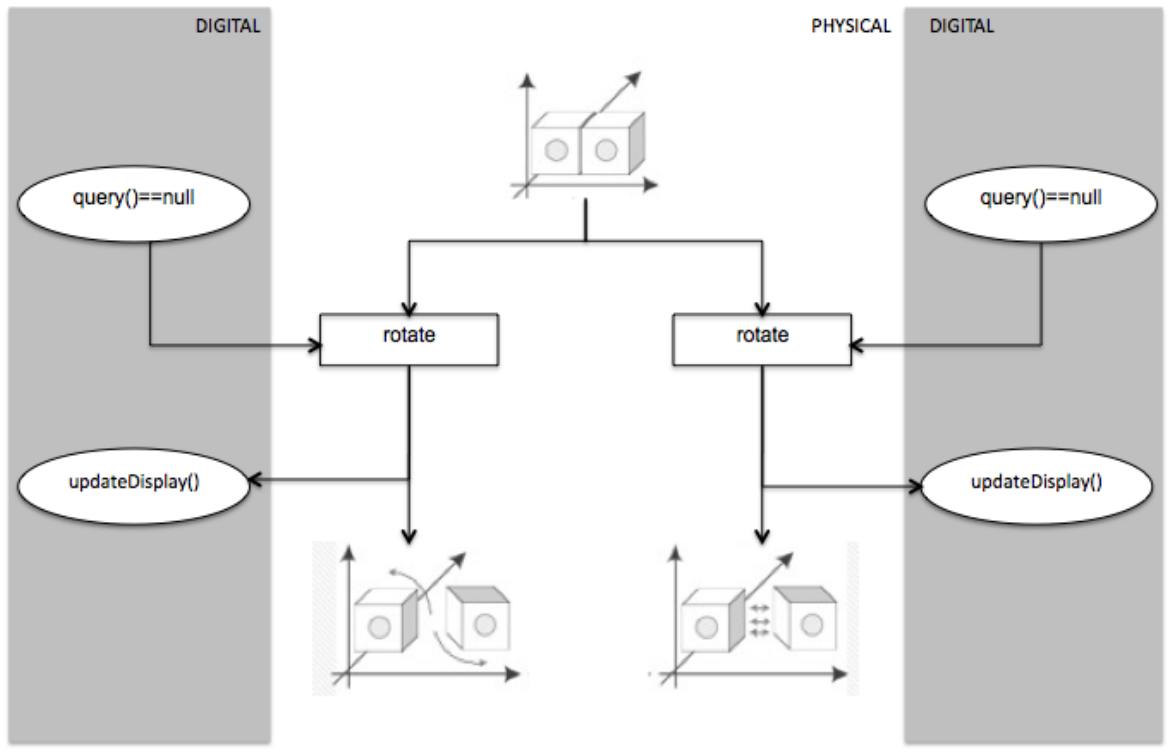

Fig. 17. Task diagram for rotating an attached block. 
3.1.3 Tangible Query Interfaces. Tangible Query Interfaces [Ullmer 2002] is a representative of the Token + Constraint category. It uses physically constrained tokens to express, manipulate and visualize parameterized database queries. Several prototypes of the Tangible Query Interfaces were built; each uses different physical token and constraints to represent query parameters. Here we refer to a prototype that employs parameter bars for representing query parameters (figure 19). Each parameter bar is equipped with a display and two sliders. By adjusting the sliders of a certain parameter bar, users can determine the upper and lower boundaries of the parameter this bar represents. When a bar is attached to the query rack, the query results are displayed. If more than one parameter bar is attached to the query rack, the system applies an AND operator to adjacent parameter and an OR operator to distant ones.
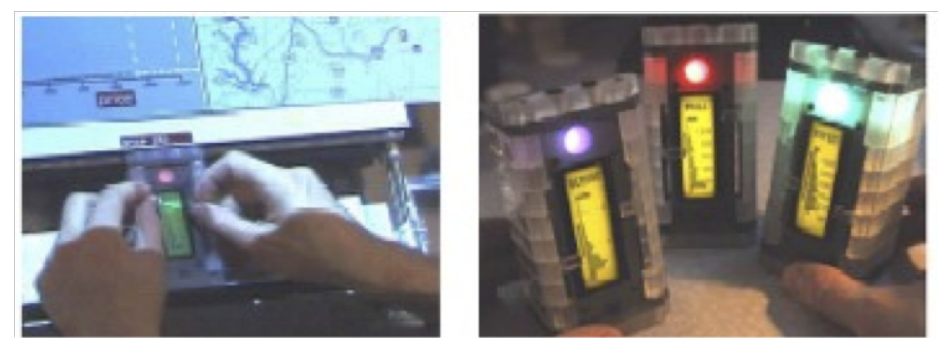

Fig. 18. Tangible Query Interfaces [Ullmer et al. 2005]

Figure 20, shows the TAC palette of a Tangible Query Interfaces prototype that uses parameter bars. The TAC palette specification highlights the recursive physical syntax this interface employs: a closer look at TAC 3 reveals that it consists of a token, a parameter bar with upper and lower sliders, constrained by a query rack. This token itself is comprised of two TACs, TAC1 and TAC2. By allowing TUI developers to easily describe nested physical structures the TAC palette provides means to examine and experiment with physical expressions of recursive grammars.

The dialogue diagram of this tangible query interface (figure 21) shows the systems two high-level states. In the first, one or more parameter bars are active (i.e. bound to a query parameter) but are not associated with the query rack. In the second high-level state, at least one parameter bar is physically associated with the query rack.

When one or more parameter bars are associated with the query rack (i.e. the system is in its second high-level state), users could slide a bar along the rack in order to alter the query. When the manipulated bar becomes adjacent to another bar, the system applies the AND operator to the two adjacent parameter bars. When it becomes distant from another parameter bar the system applies the OR operator to these bars. Figure 21, shows the task diagram for sliding a bar. Note, that the continuous manipulation slide bar generates a discrete event proximity to bar changed. Then, the system produces a digital output and updates the query display. Analyzing the task of sliding a bar using the TUIML notation highlights an 


\begin{tabular}{|c|c|c|c|c|c|c|}
\hline \multirow[t]{2}{*}{ TAC } & \multicolumn{3}{|l|}{ Representation } & \multirow{2}{*}{\begin{tabular}{|l|} 
Association \\
TAC Graphics \\
\end{tabular}} & \multicolumn{2}{|c|}{ Manipulation } \\
\hline & Variable & Token & Constraint & & Action & Response \\
\hline 1 & $\begin{array}{l}\text { Query Parameter } \\
\text { (upper bound) }\end{array}$ & Upper slider & $\begin{array}{l}\text { Bar, } \\
\text { Lower slider }\end{array}$ & & Slide & setUpperBound(0) \\
\hline 2 & $\begin{array}{l}\text { Query Parameter } \\
\text { (lower bound) }\end{array}$ & Lower slider & $\begin{array}{l}\text { Bar, } \\
\text { Upper slider }\end{array}$ & & Slide & setLowerBound() \\
\hline 3 & Query Parameter & Parameter bar & & & Add & Displays wind \\
\hline & & & Other bars & $\vec{a}$ & Slide & Hides wind \\
\hline & & & & $\stackrel{0}{\Rightarrow}$ & Remove & Updates wind \\
\hline
\end{tabular}

Fig. 19. Tangible Query Interfaces' [Ullmer et al. 2005] TAC palette

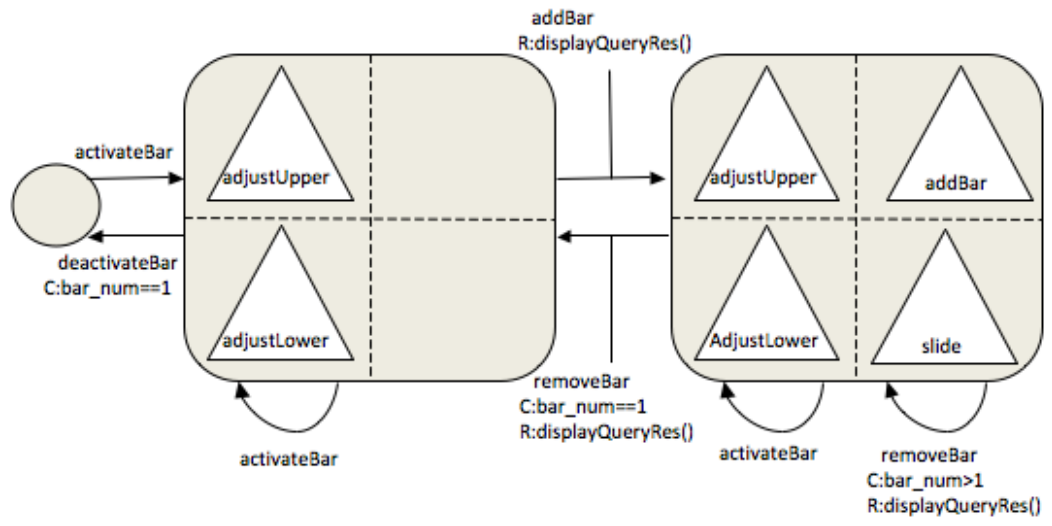

Fig. 20. Tangible Query Interfaces ${ }^{6}$ [Ullmer et al. 2005] dialogue diagram

asymmetry between user interaction (continuous) and system response (discrete). Using TUIML, TUI developers could easily model and experiment with alternative designs where the system provides continuous feedback or where the continuous manipulation of sliding a bar is replaced with a discrete action of, for example, connecting two bars.

3.1.4 discussion. The specification of benchmark TUIs demonstrates that the TUIML notation is capable of describing a broad range of interfaces. We showed that TUIML is capable of describing TUIs from the Interactive Surfaces, Constructive Assemblies and Token+Constraints categories [Ullmer 2002], that together cover a large and important subset of the tangible interaction design space. The TUIML description of each of the benchmark interfaces specified is compact and 


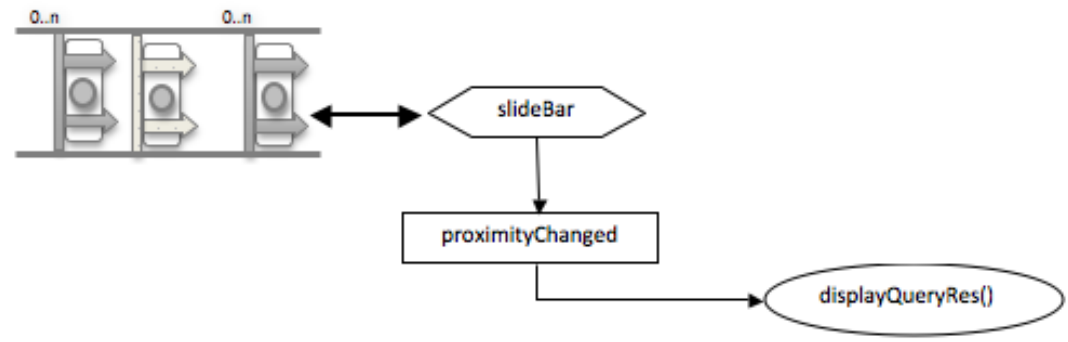

Fig. 21. Task diagram for sliding a bar within the query rack.

readable. In addition, we showed that TUIML provides TUI developers with a systematic way for considering the mapping of form and function, the use of physical syntax and the selection of meaningful interactions. Furthermore, we illustrated that TUIML can be used to compare alternative designs and to reason about parallel and continuous interactions.

\subsection{Evaluating TUIML in the Classroom}

Over four semesters, Spring 2006, Spring 2007, Summer 2007, and Spring 2008, we integrated TUIML into our TUI Laboratory course. Students used TUIML for analyzing an existing TUI, as well as in the design process of a new TUI. As most TUIs are currently developed by graduate students in research laboratories, the structure of this course and its interdisciplinary student composition provided us with an opportunity to study the use of TUIML in conditions similar to those in which many existing TUIs were developed. Following, we summarize our findings.

3.2.1 Analyzing Existing TUIs. Over these four semesters, we required students to read a TUI research paper, present it to the class and then specify it using TUIML. In addition to TUIML specifications students were required to submit a written discussion of certain aspects of the specified TUI including the use of physical syntax, parallel interaction, and treatment of boundary cases. Students selected their research paper from a list that changed over the four semesters to include recently published TUIs. Overall, the list included the nine benchmark interfaces as well as additional eight TUIs.

Overall, 28 students submitted TUIML specifications and a TUI analysis. In all semesters, we introduced TUIML in a one-hour lecture and provided an online tutorial. We did not provide an editing environment for TUIML, so students could use pencil and paper, or any computational drawing tool of their choice to create TUIML specifications. Following the submission of their specification and analysis, we asked students to fill in a questionnaire inquiring about their experience using TUIML. We found that the average time spent on TUIML specifications is 2 hours. The longest time spent on TUIML specifications was 3.5 hours. From examining students' secifications, we found that they were consistent with our specifications and captured similar TAC relationships, high-level states and actions. We also asked students how difficult was it to apply TUIML to their TUI of choice (on a scale 
of 1 to 5 , where 5 is very difficult) and how confident they are that they correctly identified TUIML elements (on a scale of 1 to 5 , where 5 is very confident). Table 2 summarizes students responses to those questions regarding the specification of our benchmark interfaces. The instances column in table 2 refers to by how many students an interface was specified. It is important to note that while this data do not lend itself to statistical analysis and hence cannot be generalized, it shows that students from a variety of backgrounds understood the concepts of TUIML and successfully completed the TUIML specification of an existing TUI with only minimal training and without exceptional difficulties.

Finally, we asked students what they liked and disliked about TUIML, and in what ways TUIML could be improved. Following are selected students' responses: What students liked: "It (TUIML) forced me to really understand all aspects of the TUI in detail" (Spring 2006). "The graphical rather than textual description, made it easier to visually conceptualize the system in different states" (Spring 2007). "It helped me think about aspects of the TUI design that I didnt consider before such as having multiple users interacting with the system in parallel" (Spring 2007). "I like that it distills complicated systems into much simpler elements that can be used as a framework for comparison" (Spring 2008) What students disliked: "I was a little confused sometimes in the dialogue diagram about what should be considered an action and what is a transition" (Spring 2006). "It does not support specifying changing users, since different users change how data is interpreted I feel it is important"(Summer 2007). "With passive user interfaces, TUIs without a continuous link to an underlying technology, a lot of the meaningful activity that is not computationally interpreted is lost in the description " (Spring 2007). Students' suggestions for improvements: " expanding the pictorial notation will make TUIML easier to use and read", "I would change the symbols used for tokens, they are a bit ambiguous" (Spring 2007, Senseboard). "attributes of tokens are hard to represent, how do you specify a range of valuesi‘" (Spring 2006). "introduce error states in the dialogue diagram (Spring 2008).

Table II. Responses to selected questions from the TUIML questionnaire regarding the specification of benchmark interfaces.

\begin{tabular}{|l|l|l|l|}
\hline Interface & Instances & Difficulty & Confidence \\
\hline Urp [Underkoffler and Ishii 1999] & Used as an example & & \\
\hline Designers' Outpost [Klemmer et al. 2001] & 3 & 2 & 3.7 \\
\hline Senseboard [Jacob et al. 2002] & 3 & 2 & 4 \\
\hline TVE [Zigelbaum et al. 2007] & 3 & 2 & 4 \\
\hline Navigational Blocks [Camarata et al. 2002] & 2 & 3 & 4 \\
\hline Tern [Horn and Jacob 2007] & 2 & 2 & 4.3 \\
\hline Marble Answering Machine [Crampton-Smith 1995] & Used as an example & & \\
\hline Tangible Query Interfaces [Ullmer 2002] & 2 & 2 & 3.5 \\
\hline Media Blocks [Ullmer 2002] & 2 & 3.5 & 3.2 \\
\hline
\end{tabular}


3.2.2 Using TUIML in the Design Process of TUIs. Between Spring 2006 and Spring 2008, 11 groups of students used TUIML in the design process of their TUI laboratory course project. We asked students to submit their TUIML specifications when their conceptual design was complete and then again with the final documentation of their project. Following the completion of their project, we asked students to answer a questionnaire about their experience using TUIML. All student groups completed and submitted their TUIML specifications without further help from us. The average time that took the groups to complete the first round of specifications was 1.5 hours (as reported by students). The longest time for completion was reported as 3 hours.

We also asked students about how the TUIML modeling process benefitted their design process. Following are samples of students' responses: "TUIML helped us focus more clearly on how our system would work. By abstracting away the implementation aspect of what we were doing, we were able to design a system that had fundamentally sound interaction concepts without being distracted by how we are going to actually implement them", "Once we had the TUIML model it was much easier to divide the work and progress ", "TUIML gave us a clear abstraction of how we wanted the users to interact with the system. This allowed us to focus on designing those pieces well for our prototype, rather than spending a lot of time on parts that weren't as crucial to the actual user interaction", "TUIML made me look at the relationships between our tokens in a new way because I started to consider how the user would interact with the system and see what implicitly fits together".

\subsection{Discussion}

The results we presented here have some limitations. First, the evaluation of TUIML was conducted within a classroom setting, students were required to learn TUIML in order to complete their assignments; thus, we may anticipate some bias in students' responses to the questionnaire. Second, students' experience with TUIML may vary between the semesters due to changes in the language itself and in the online tutorial. Therefore, these evaluation results may only be qualified as tentative. However, these results do demonstrate that TUIML is capable of describing a broad range of existing and new TUIs, across the space of the tangible interaction design space. Furthermore, our evaluation shows that TUIML has a relatively low threshold. Students from a variety of backgrounds successfully completed the TUIML specifications of an existing TUI with only minimal training. The use of TUIML specifications in the design process of new TUIs, shows that students understood the concepts and possibilities of TUIML and put them to good use. Also, the effort required from students to complete TUIML specifications was not greater then the effort required to read a scientific paper, create a storyboard, or write a natural language interface description. The specification of benchmark interfaces as well as students' feedback provide some evidence for the utility of TUIML: exploring and defining relationships between physical interaction objects, considering parallel interaction, comparing alternative designs, highlighting boundary cases, and using specifications as a basis for communication. Finally, most of the students suggestions for improvement were implemented in the current version of TUIML. 
Having demonstrated that TUIML is capable of describing a broad range of interfaces, has a low-threshold, and provides utility, next we show that TUIML specifications could be semi-automatically converted into concrete TUI implementation by a TUIMS.

\section{TOWARD A TANGIBLE USER INTERFACE MANAGEMENT SYSTEM}

A Tangible User Interface Management System (TUIMS), draws upon User Interface Management System (UIMS) research to provide support for editing TUIML specifications, translating them into concrete implementations, and managing the interaction with a variety of interaction devices at run-time. Similarly to a UIMS, a TUIMS supports the concept of "dialog independences" [Hartson and Hix 1989], the separation of all user interface related code from the application code. This allows changes to be made in the TUI design without affecting the application code. Thus, it supports iterative prototyping and exploration of new technologies. Following, we lay the foundation for the development of a TUIMS. We present a top-level architecture for a TUIMS, and describe a proof-of-concept TUIMS prototype.

\subsection{TUIMS Architecture}

In a seminal paper, Foley and Wallace suggested to decompose the user interface design into semantic, syntactic and lexical levels [Foley and Wallace 1974]. This top down approach allows useful modularity during the design of user interfaces and serves as a foundation for various user interface software tools including for the software architecture of many UIMSs [Olsen 1992]. This decomposition is also found in our design of the TUIMS architecture (see figure 23) that draws from UIMS architecture models such as the early Seeheim model [Olsen 1992], and the later Arch/Slinky [uim 1992] and PAC-Amodeus [Nigay and Coutaz 1991] models.

The TUIMS captures the semantic level of a TUI in the application code. The syntactic level of a TUI consists of a description of the logical physical interaction objects and the manipulation actions users may perform upon them. For each manipulation action it provides the context in which it may be performed which in turn determines which functions to invoke. The syntactic level is captured in the TUIML models and is translated into the TUIMS model component. Modeling tools can support the development of the TUIML models.

The lexical level of a TUI deals with device discovery and communication as well as with the precise mechanism by which a user specifies the syntax. A Lexical Handler is a TUIMS component that is responsible for the communication and user interaction with a set of devices mediated by a particular implementation technology. A TUIMS may contain several Lexical Handlers. Physical and graphical toolkits can be used in the implementation of a lexical handler. The mapping of low-level input events to syntactic events is performed by the Lexical Manager.

The Dialog Manager is driven automatically or semi-automatically from TUIML specifications. It controls the logical interaction objects and is responsible for invoking application functions in response to manipulation actions as well as for firing physical output events in responses to changes in the internal state of the application. The communication between the Dialog Manager and the Application logic is performed via the semantic interface. The communication between the Dialog Manager and the Lexical Manager is performed via the syntactic interface while the

ACM Journal Name, Vol. V, No. N, June 2009. 
communication between the Lexical Manger and the lexical handlers is performed via lexical interfaces. This architecture is intended to facilitate the development of technologically portable TUI systems by allowing TUIMS users to modify and add Lexical Handlers that support a variety of implementation technologies.

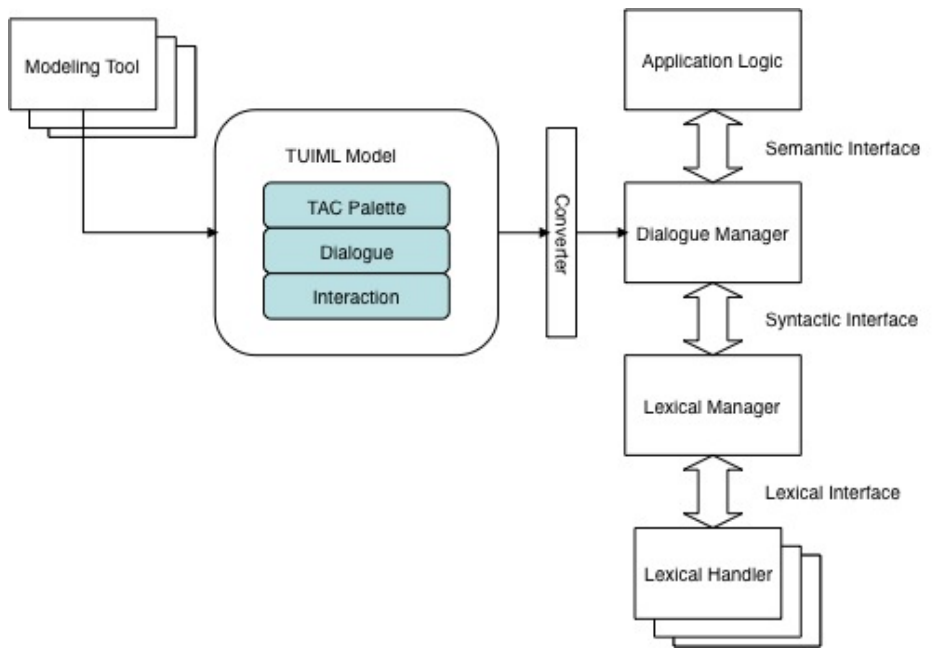

Fig. 22. The TUIMS architecture.

\subsection{Proof-of-Concept Prototype}

In order to investigate and demonstrate the feasibility of implementing a TUIMS, we developed a proof-of-concept TUIMS prototype. Our prototype is not a complete implementation of a TUIMS, rather it serves as a working model of a TUIMS back-end that implements the TUIMS top-level architecture. It receives XMLcompliant TUIML specifications as input, semi-automatically translates them into a concrete TUI implementation, and serves as a run-time environment that manages the communication and interaction with different implementation technologies. We used this prototype to successfully program several existing TUIs including the Marble Answering Machine [Crampton-Smith 1995], a reduced functionality Urp [Underkoffler and Ishii 1999], and the Marble Track Audio Manipulator [Bean et al. 2008]. We chose Java as an implementation language for our prototype because of its extensive library support, platform independence, the potential to integrate TUIML with emerging TUI toolkits (e.g. [Hartmann et al. 2006; Hartmann et al. 2007; Klemmer et al. 2004]), and novice software developers fluency. However, platform independence comes at a performance cost. For the development of most TUIs we estimated that ease of technology integration and rich library support gains are more valuable than performance. Following we describe this prototype.

4.2.1 Input Abstraction and Event Generation. The input layer of a TUIMS consists of a set of lexical handlers that are responsible for the communication and user interaction with a set of devices mediated by a particular implementation 
technology. We developed three lexical handlers supporting implementation technologies that are often used in the development of TUIs: RFID, microcontrollers (Handy Board microcontroller) and arcade controllers (I-PAC Arcade Controller). Following, we briefly describe each of these lexical handlers.

RFIDHandler. This lexical handler communicates with an FEIG OBID i-scan RFID Reader. It polls the RFID reader through a serial connection at regular intervals and generates events when tags are added or removed from the readers field of view. It generates tagAdded and tagRemoved events, both contain two strings: tag ID and reader ID. The RFIDHandler can also read/write data to individual tags. It is implemented using the Java Communications API.

HBDHandler. The Handy Board microcontroller was designed for experimental robotics projects, but it also serves a large number of embedded control applications. The Handy Board is programmed with Interactive $\mathrm{C}$, a subset of the $\mathrm{C}$ programming language. The HBDHandler consists of two parts. The first is a Java program that communicates with the Handy Board over a serial connection. It fires an event when new input is read through the serial connection and generates three types of events: CircuitOn and CircuitOff which, represent a change in a digital sensor and contain port ID, and AnalogueChanged which, represents a change in the value read by an analogue sensor and contains a port ID and a value. The HBDHandler also enables to send a command to one of the Handy Board motor ports. The second part of this lexical handler is an Interactive $\mathrm{C}$ program that runs on the Handy Board and communicates with its serial connection.

IPACHandler. The I-PAC Arcade Controller is a USB keyboard emulator that simplifies input acquisition from switches, arcade buttons, joysticks, etc. A WIN32 firmware programmer enables to map input ports to standard keyboard keys. The IPACThread implements the Java KeyListener interface to receive input from the I-PAC controller. It fires CircuitOn and CircuitOff events that contain port ID.

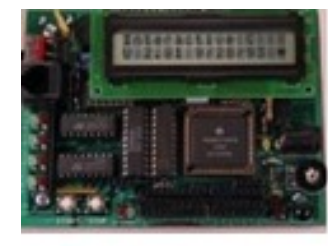

Handy Board

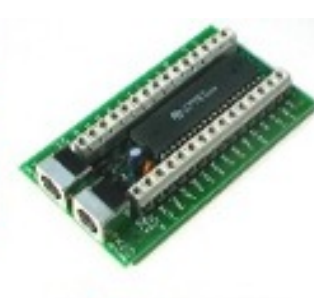

I-PAC arcade controller

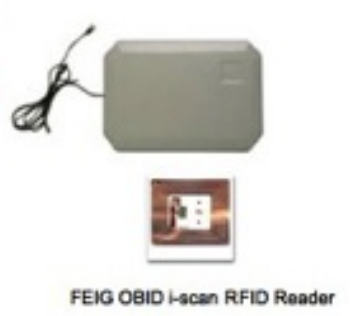

Fig. 23. TUI implementation technologies supported by our proof-of-concept TUIMS prototype.

Each of these lexical handlers implements the LexicalHandler interface. Unlike a GUI toolkit that typically supports one mouse and one keyboard, several lexical handlers can run in parallel supporting multiple input devices and multiple implementation technologies. Figure 25, shows a class diagram of the TUIMS 
event handling mechanism. Once a lexical handler fires an event it is dispatched by a subclass of a TUIMLEventProducer, which is the class responsible for mapping low-level events to TUIML events. TUIML events include TokenAdded, TokenRemoved, TokenPropertyChanged and TokenUpdated. While these events are consistent across the different implementation technologies, each implementation technology may provide different information. For each lexical handler there is a corresponding producer class that dispatches low-level events and generates TUIML events. The separation of input acquisition from event dispatch and generation allows users to modify a particular TUIMLEventProducer instance. A complete TUIMS should provide users with an easy way to map low-level events to TUIML events for example by using a form-based interface to edit a particular TUIMLEventProducer. The LexicalManager is responsible for instantiating TUIMLEventProducer instances and handling exceptions that result from hardware configuration. Finally, the DialogueManager implements the TUIMLEventListener interface and dispatches TUIML events. It responds to events by instantiating Token and TAC objects as well as by calling application logic methods and updating the application state.

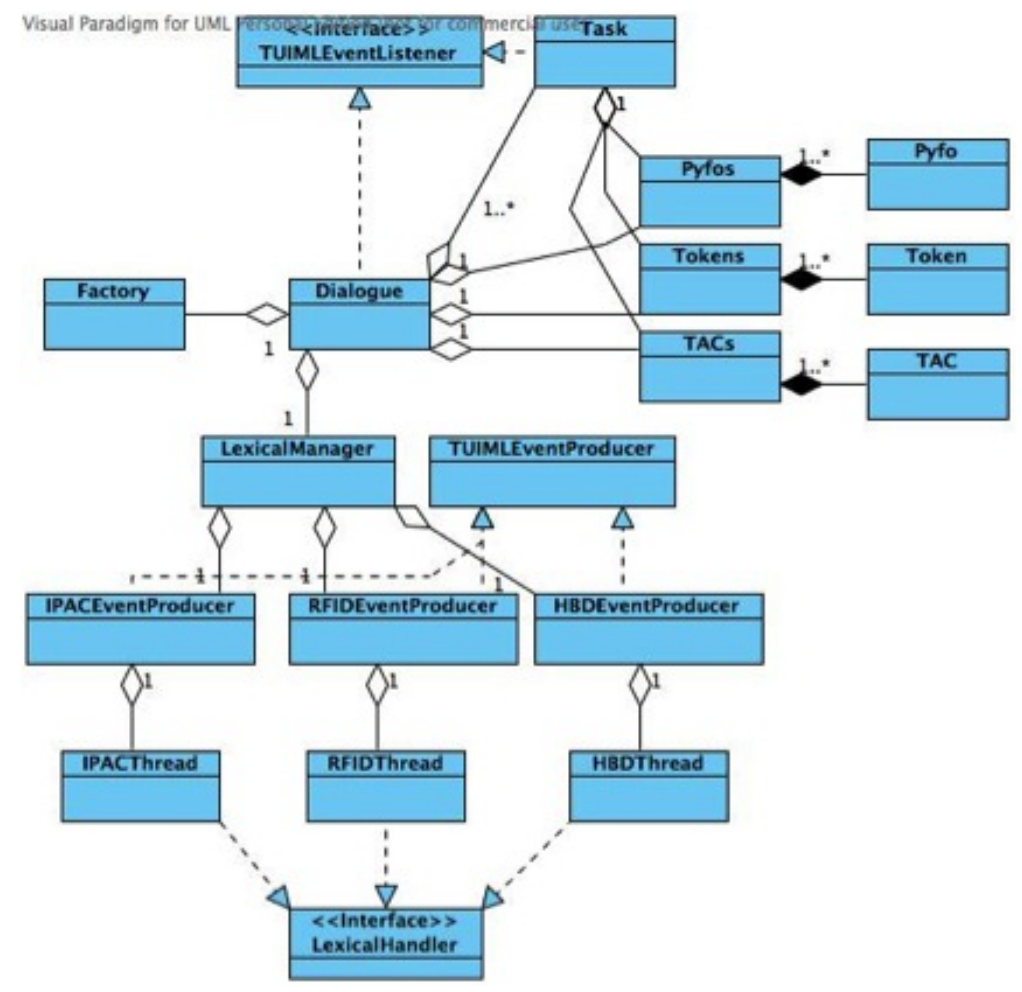

Fig. 24. A class diagram of the TUIMS event handling mechanism 
4.2.2 Control Flow. The basic flow of control in an application programmed with TUIMS is that the DialogueManager instantiates the LexicalManager and listens to TUIML events while keeping track of instantiated TUIML objects (i.e. pyfo, token and TAC objects) and of the current application state. It also contains a Factory object that is responsible for instantiating Pyfo, Token and TAC objects. When a TUIML event is dispatched the DialogueManager fires a transition according to the event type and the current state of an application. The Factory class is used for creating certain Token or TAC objects as a response to a particular transition. When the system enters a new state the DialogueManager launches a thread for each of the tasks available for users to perform when the system is in that particular state. Each of these threads implements a TUIMLEventListener interface and responds to TUIML events according to the pre and post conditions specified in the TUIml interaction element. The task threads and the DialogManager both track the physical state of the system. When a task is no longer available the DialogManager stops its thread.

4.2.3 Code Generation. Finally, we implemented a TUIml2JavaCompiler that generates Java code from XML-compliant TUIML specifications. Our TUIml2JavaCompiler works as following:

(1) Creates a class for each specified tac and token elements. A class for a tac element extends the base class TAC, a class for a token element extends the base class Token.

(2) Creates a Factory class with create and destroy methods for each TAC or Token instances.

(3) Creates an instance of a Task class for each specified task element.

(4) Instantiates a LexicalManager that contains a reference to a LexicalHandler and TUIMLEventProducer for each implementation technology discovered by the TUIml2JavaCompiler.

(5) Creates a DialogueManager class that contains:

- reference to a lexical manager.

-reference to an application logic.

- flag for each task element that is specified in the TUIML interaction element.

- reference to a Task instance created for each task element specified.

- private init method that instantiates each of the specified pyfo and token elements.

- private initTasks method that initialized each Task instance.

- private setTasksFlags method that sets the task flags according to the current application state.

- private fireTransition method that fires each of the specified transitions.

While the compiler generates a skeleton of a concrete Java implementation as described above, currently, a programmer is still required to manually program methods. Nevertheless, our implementation of the TUIml2JavaCompiler demonstrates that semi-automatic code generation from TUIml specification is feasible.

ACM Journal Name, Vol. V, No. N, June 2009. 


\subsection{Summary}

We introduced the concept of a TUIMS, that draws upon UIMS research [Olsen 1992] to provide support for editing high-level specifications, translating them into concrete implementations, and managing the interaction with a variety of interaction devices at run-time. We presented a top-level architecture for a TUIMS and described a proof-of-concept TUIMS prototype that demonstrates input abstraction and event generation, control flow, and semi-automatic code generation. We believe that other aspects of TUIMS implementation such as a visual editing environment, additional lexical handlers, and an integrated development environment could be implemented by additional programming effort using well-known techniques.

\section{RELATED WORK}

\subsection{User Interface Management System}

In the early 1980s, the concept of a user interface management system (UIMS) was an important focus area for the then-forming user interface software research community [Myers et al. 2000]. The goals of UIMS research were to: simplify the development of user interfaces by shielding developers from implementation details, to promote consistency across applications, and to facilitate the separation of user interface code from application logic. A UIMS implemented the syntactic and lexical levels of the user interface and conducted the interaction with the user [Olsen 1992]. It allowed designers to specify interactive behavior using a UIDL. This specification was then automatically translated into an executable program or interpreted at run time. The choice of UIDL model and methods was a key ingredient in the design and implementation of the UIMS [Olsen 1992]. Many systems used techniques borrowed from formal languages or compilers for dialogue specification, for example: state transition diagrams and advanced state machine models [Newman 1968; Jacob 1986; Olsen 1984], and parsers for context-free grammars (e.g. [Olsen and Dempsey 1983]). Others used event-based specification to specify the interface responses to IO events (e.g.[Goodman 1987]).

Although UIMS offered a promising approach for simplifying the development of UIs, this approach has not worked out well in practice [Myers et al. 2000]. While UIMS tried to isolate the decision of how the interaction will look and feel from the designers and offer a consistent look and feel across applications, designers felt that the control of this low level pragmatics is important. Furthermore, most UIDLs had a high-threshold, they required a substantial learning effort to successfully specify an interface. Finally, the standardization of UIs on the desktop paradigm made the need for abstractions from the input/output devices mostly unnecessary. Subsequently, in the last decade, some of the challenges facing the developers on next generation user interfaces are similar to those that faced GUI developers in the early 1980. Thus, as part of the user interface software research community effort to address these difficulties, the concept of a UIDL reemerged as a promising approach.

\subsection{Emerging User Interface Description Languages}

Several UIDLs, most of them XML-based, have been developed in recent years in order to accomplish the following goals [Luyten et al. 2004]: capturing the require- 
ments for a user interface as an abstract definition that remains stable across a variety of platforms, enabling the creation of a single user interface design for multiple devices and platforms, improving the reusability of a user interface, supporting evolution and extensibility of a user interface, and enabling automated generation of user interface code. TUIML shares most of the above goals with emerging UIDLs, while aiming at developing TUIs rather than muti-platform interfaces. Several UIDLs that support the generation of multi-platform and multi-modal user interfaces from a single abstract description, inspired the design of TUIML: The ARTStudio [Calvary et al. 2001], is a tool that supports the design process of plastic user interfaces by exposing several models to the designer: task, concepts, platform, interactors, abstract and concrete user interfaces. The User Interface Markup Language (UIML) [Ali et al. 2004], is an XML-based UIDL that provides a device independent method for user interface design. UIML specifications first define the abstract types of user interface components and the code to execute when events occur, and are then further refined into a specific user interface. The USer Interface eXtensible Markup Language (USIXML) [Limbourg et al. 2004] allows the specification of task, domain, presentation, and context-of-use models while providing a substantial support for describing relationships between these models. USIXML supports the generation of multi-modal user interfaces. Finally,Teresa XML [Paternò and Santoro 2002] enables the specification of task models, composition of interactors and a user interface dialogue. An extensive set of tools supports the development of concrete user interfaces from Teresa XML specifications.

While we have witnessed an extensive development of UIDLs for the development of user interfaces for multiple platforms and contexts, only few UIDLs address the development of next generation user interfaces. Jacob et al. presented a software model, a language and a development environment - PMIW, for describing and programming non-WIMP virtual environments [Jacob et al. 1999]. Their model addresses the continuous aspect of non-WIMP interaction explicitly by combining a data-flow component for describing continuous relationships with an event-based component for specifying discrete interactions. The model also supports parallel interaction implicitly because it is simply a declarative specification of a set of relationships that are in principle maintained simultaneously. The model abstracts away many of the details of specific interaction devices and treats them only in terms of the discrete events they produce and the continuous values they provide. Similarly to the PMIW approach, TUIML abstracts implementation details and describes the behavior of a TUI using two communicating components. However, TUIML also provides means for explicitly specifying parallel interaction, and for describing relations between physical interaction objects. InTML [fig ] and Chasm [Wingrave and Bowman 2008] are more recent UIDLs for describing VR applications. Similarly to PMIW, they are platform and toolkit independent and targets non-programmers.

\subsection{Toolkits for Physical Interaction}

In the last years, the research community has developed several tools to support physical computing including Phidgets [Greenberg and Fitchett 2001], iStuff [Ballagas et al. 2003], Exemplar [Hartmann et al. 2007] and Papier-Mache [Klemmer et al. 2004]. The major advantage of these toolkits is that they lower the threshold 
for implementing fully functional TUI prototypes by hiding and handling low-level details and events. However, they fall short of providing a comprehensive set of abstractions for specifying and discussing tangible interaction. In section 1.3 .3 we discuss toolkits for physical interaction in further detail.

\section{CONCLUSIONS AND FUTURE WORK}

This paper contributes TUIML, a high-level UIDL, that supports the design and implementation of TUIs by providing TUI developers from different backgrounds means for specifying, discussing, and programming tangible interaction. There are three distinct elements to this contribution: a visual specification technique that is based on Statecharts and Petri Nets, an XML-compliant language that extends this visual specification technique, as well as a proof-of-concept prototype of a TUIMS that semi-automatically translates TUIML specifications and manages the communication and interaction with a variety of interaction devices at run-time. The evaluation of TUIML shows that it is capable of describing a broad range of existing and new TUIs and has a relatively low-threshold. It also demonstrates that TUIML specifications alleviate TUI development challenges while requiring an effort that is not greater than required by existing tools and techniques. Finally, the TUIMS proof-of-concept prototype presented here demonstrate that TUIML specifications could also be semi-automatically converted to a concrete implementation.

Currently, we are extending TUIML to support whole-body interaction by specifying expressive movement. We also seek to integrate TUIML with standard specification languages and with existing toolkits for tangible interaction.

\section{ACKNOWLEDGMENTS}

We thank Judith Stafford, Samuel Gayer, Harriet Fell, Ray Jackendoff, Michael Horn, Audrey Girouard, Erin Solovey, and Jamie Zigelbaum for their valuable feedback on this work. We also thank Tufts' students who provided invaluable support for this work: Amy Van Holten, Katelyn Mann and Jeff Livingston. Finally, we thank our students at the TUI laboratory course for sharing their enthusiasm, creativity and insights. This material is based in part upon work supported by the National Science Foundation under Grant Numbers EHR-0227879 and IIS-0414389.

\section{REFERENCES}

1992. A metamodel for the runtime architecture of an interactive system: the uims tool developers workshop. SIGCHI Bull. 24, 1, 32-37.

Ali, M. F., Perez-Quinones, M. A., And Abrams, M. 2004. Building multi-platform user interfaces with uiml. In Multiple User Interfaces, S. A. and J. H., Eds. John Wiley and Sons, Chichester, UK, 95-116.

Appert, C. And Beaudouin-Lafon, M. 2006. Swingstates: Adding state machines to the swing toolkit. In UIST'06: Symposium on User Interface Software and Technology. ACM Press, Montreux, Switzerland.

Ballagas, R., Ringel, M., Stone, M., And Borchers, J. 2003. istuff: A physical user interface toolkit for ubiquitous computing environments. In CHI'03: Human Factors in Computing Systems. ACM Press.

Bastide, R. And Palanque, P. A. 1990. Petri net objects for the design, validation and prototyping of user-driven interfaces. In INTERACT '90. North-Holland Publishing Co. 
Bean, A., Siddiqi, S., Chowdhury, A., Whited, B., Shaer, O., and Jacob, R. J. K. 2008. Marble track audio manipulator (mtam): a tangible user interface for audio composition. In TEI'08. ACM.

Blackwell, A., Edge, D., Dubuc, L., Rode, J., Stringer, M., And Toye, E. 2005. Using solid diagrams for tangible interface prototyping. IEEE Pervasive Computing Oct-Dec, 18-21.

Brooks, F. P. 1987. No silver bullet essence and accidents of software engineering. Computer 20, 4, 10-19.

Calvary, G., Coutaz, J., And Thevenin, D. 2001. A unifying reference framework for the development of plastic user interfaces. In EHCI '01: Proceedings of the 8th IFIP International Conference on Engineering for Human-Computer Interaction. Springer-Verlag.

Camarata, K., Do, E. Y.-L., Johnson, B. R., And Gross, M. D. 2002. Navigational blocks: navigating information space with tangible media. In IUI '02. ACM.

Crampton-Smith, G. 1995. The hand that rocks the cradle. I.D May/June, 60-65.

Foley, J. D. and Wallace, V. L. 1974. The art of natural graphic man-machine conversation. Proceedings of the IEEE 62, 4, 462-471.

Goodman, D. 1987. The Complete HyperCard Handbook. Bantam Books, New York.

Greenberg, S. And Fitchett, C. 2001. Phidgets: easy development of physical interfaces through physical widgets. In UIST '01. ACM, 209-218.

Harel, D. 1988. On visual formalisms. Comm. ACM 31, 5, 514-530.

Hartmann, B., Abdulla, L., Mittal, M., and Klemmer, S. R. 2007. Authoring sensor-based interactions by demonstration with direct manipulation and pattern recognition. In $\mathrm{CHI}$ 'O\%. ACM.

Hartmann, B., Klemmer, S. R., Bernstein, M., Abdulla, L., Burr, B., Robinson-Mosher, A., AND GeE, J. 2006. Reflective physical prototyping through integrated design, test, and analysis. In UIST '06. ACM.

Hartson, H. R. And Hix, D. 1989. Human-computer interface development: Concepts and systems for its management. Computing Surveys 21, 1, 5-92.

Horn, M. S. AND JACOB, R. J. K. 2007. Tangible programming in the classroom with tern. In CHI '07: extended abstracts on Human factors in computing systems. ACM.

Hornecker, E. And Buur, J. 2006. Getting a grip on tangible interaction: A framework on physical space and social interaction. In CHI'06: Human Factors in Computing Systems. ACM, Montreal, Canada, 437-446.

IshiI, H. AND Ullmer, B. 1997. Tangible bits: towards seamless interfaces between people, bits and atoms. In $C H I$ ' $97 . \mathrm{ACM}, 234-241$.

Jacob, R. J., Girouard, A., Hirshfield, L. M., Horn, M. S., Shaer, O., Solovey, E. T., And Zigelbaum, J. 2008. Reality-based interaction: a framework for post-wimp interfaces. In $C H I$ '08: Proceeding of the twenty-sixth annual SIGCHI conference on Human factors in computing systems. ACM, New York, NY, USA, 201-210.

JАCOB, R. J. K. 1986. A specification language for direct manipulation user interfaces. ACM Transactions on Graphics 5, 4, 283-317.

Jacob, R. J. K., Deligiannidis, L., And Morrison, S. 1999. A software model and specification language for non-wimp user interfaces. ACM Transactions on Computer-Human Interaction 6, 1, 1-46.

Jacob, R. J. K., Ishit, H., Pangaro, G., and Patten, J. 2002. A tangible interface for organizing information using a grid. In CHI '02: Proceedings of the SIGCHI conference on Human factors in computing systems. ACM, New York, NY, USA, 339-346.

Janssen, C., Weisbecker, A., And Ziegler, J. 1993. Generating user interfaces from data models and dialogue net specifications. In CHI '93: Proceedings of the INTERACT '93 and CHI '93 conference on Human factors in computing systems. ACM, 418-423.

Klemmer, S. R., Li, J., Lin, J., And Landay, J. A. 2004. Papier-mache: toolkit support for tangible input. In CHI '04: Proceedings of the SIGCHI conference on Human factors in computing systems. ACM, New York, NY, USA, 399-406.

ACM Journal Name, Vol. V, No. N, June 2009. 
Klemmer, S. R., Newman, M. W., Farrell, R., Bilezikjian, M., and Landay, J. A. 2001. The designers' outpost: a tangible interface for collaborative web site. In UIST '01: Proceedings of the 14th annual ACM symposium on User interface software and technology. ACM, New York, NY, USA, 1-10.

Limbourg, Q., V, J., Michotte, B., Boulllon, L., And Lpez-Jaquero, V. 2004 . Usixml: a language supporting multi-path development of user interfaces. Springer-Verlag, 11-13.

Luyten, K., Abrams, M., Vanderdonckt, J., And Limbourg, Q. 2004. Developing user interfaces with xml: Advances on user interface description languages. an AVI'04 workshop.

Myers, B., Hudson, S. E., And Pausch, R. 2000. Past, present, and future of user interface software tools. ACM Transactions on Computer-Human Interaction 7, 1, 3-28.

Newman, W. M. 1968. A system for interactive graphical programming. In AFIPS '68 (Spring): Proceedings of the April 30-May 2, 1968, spring joint computer conference. ACM, New York, NY, USA, 47-54.

Nigay, L. And Coutaz, J. 1991. Building user interfaces: organizing software agents. In Esprit '91 Conference Proceedings, ACM, Ed.

Olsen, D. R. 1984. Push-down automata for user interface management. ACM Transactions on Graphics 3, 3, 177-203.

Olsen, D. R. 1992. User Interface Management Systems: Models and Algorithms. Morgan Kaufmann, San Francisco. ISBN 1-55860-220-8.

Olsen, D. R. And Dempsey, E. P. 1983. Syngraph: A graphical user interface generator. Computer Graphics 17, 3, 42-50.

Paternò, F. And SAntoro, C. 2002. One model, many interfaces. In CADUI. 143-154.

Petri, C. 1962. Communikation mit automaten. Ph.D. thesis, University of Bonn.

Plaisant, C. 2004. The challenge of information visualization evaluation. In AVI '04: Proceedings of the working conference on Advanced visual interfaces. ACM, New York, NY, USA, 109-116.

Shaer, O., Leland, N., Calvillo-Gamez, E. H., And Jacob, R. J. K. 2004. The tac paradigm: Specifying tangible user interfaces. Personal and Ubiquitous Computing 8, 5, 359-369.

Truong, K. N., Hayes, G. R., And Abowd, G. D. 2006. Storyboarding: an empirical determination of best practices and effective guidelines. In DIS '06: Proceedings of the 6th conference on Designing Interactive systems. ACM, New York, NY, USA, 12-21.

Ullmer, B. A. 2002. Tangible interfaces for manipulating aggregates of digital information. Ph.D. thesis, Massachusetts Institute of Technology. Doctoral Dissertation.

Underkoffler, J. AND IshiI, H. 1999. Urp: A luminous-tangible workbench for urban planning and design. In CHI'99: Human Factors in Computing Systems. ACM Press, 386-393.

Weber, M. And Kindler, E. 2003. The petri net markup language. In Petri Net Technology for Communication-Based Systems. Vol. 2472/2003. Springer Berlin / Heidelberg, 124-144.

Wingrave, C. A. And Bowman, D. A. 2008. Tiered developer-centric representations for 3d interfaces: Concept-oriented design in chasm. In VR. 193-200.

Zigelbaum, J., Horn, M. S., Shaer, O., and Jacob, R. J. K. 2007. The tangible video editor: collaborative video editing with active tokens. In TEI '07: Proceedings of the 1st international conference on Tangible and embedded interaction. ACM, 43-46. 